\title{
Dynamics of an anchored Toom interface
}

\author{
B Derrida $\uparrow \S$, J L Lebowitz $\ddagger \|$, E R Speer $\ddagger$ and H Spohn $\dagger \uparrow$ \\ $\dagger$ School of Mathematics\#, Institute for Advanced Study, Princeton, NJ $0 \overline{8} 540$, USÁ \\ ¥Department of Mathematics, Rutgers University, New Brunswick, NJ 08903, USA
}

Received 3 June 1991

\begin{abstract}
At a sufficiently low noise level the two-dimensional Toom model (North East Center majority vote with small errors) has two stationary states. We study the statistical properties of interfaces between these phases, with particular attention to a stationary interface maintained in the third quadrant by mixed +- boundary conditions. The fluctuations in this interface are found numerically to be much smaller than in equilibrium interfaces; they have exponents $\frac{1}{4}$ or $\frac{1}{3}$, depending on the symmetry, rather than $\frac{1}{2}$. The correlations exhibit long-range behaviour reminiscent of self-organized criticality. We construct several approximate theories of the interface which reproduce this behaviour, at least qualitatively. The most accurate of these leads to a novel nonlinear partial differential equation for the asymptotic probability distribution of the fluctuations along the interface.
\end{abstract}

Dedicated to Michael Fisher on the occasion of his sixtieth birthday.

\section{Introduction}

There is much current interest in the structure of states of lattice systems obtained as stationary measures for various kinds of stochastic dynamics. These states typically do not correspond to equilibrium Gibbs ensembles with any reasonable interactions. They can exhibit behaviour quite different from that familiar for equilibrium systems, e.g. power law decay of correlations corresponding to generic scale invariance (or self-organized criticality) [1-3] and coexistence of multiple phases, i.e. non-ergodicity, under a wide range of perturbations of the dynamics [6].

Examples of non-equilibrium stationary states are those obtained from various types of majority rule dynamics used as models for pattern recognition and for error-tolerant computations. Models of this type include cellular automata with noise: systems in which spins are updated stochastically at integer times, simultaneously at all sites of some regular lattice. These models were first investigated in detail in the Soviet literature of the late 1960 s and early 1970s [4]. (Toom et al [4] provide a recent review of Soviet work.) They are now generally referred to as stochastic or probabilistic cellular automata (for recent physics-oriented work see [5]), and may be considered to include deterministic automata as special limits.

$\S$ Permanent address: SPT, CEN Saclay, F91191 Gif-sur-Yvette, France.

|| Also at Department of Physics, Rutgers. Supported in part by NSF Grant DMR 89-18903.

I] Permanent address: Theoretische Physik, Universität München, Theresienstrasse 37,8 München 2, Federal Republic of Germany.

\# Supported in part by NSF Grant DMS 86-10730. 
A particularly interesting probabilistic cellular automaton is the Toom model [6]. This is the only non-equilibrium model without traps we know of for which one can prove rigorously the existence of a phase transition as the amount of noise added to a deterministic majority rule is changed. There is a unique stationary state-the system is ergodic - at high noise levels, and there are (at least) two stationary states, so that the system is not ergodic, at low noise. The low-noise phases are stable against arbitrary small changes in the dynamical rules, in striking contrast to coexisting phases under dynamics with detailed balance, where a small change generically moves the system away from the line of phase coexistence.

We shall consider here the Bennett-Grinstein version (the NEC or North East Center majority rule) of the two-dimensional (2D) Toom model [7]: the system consists of Ising spins $\left(\sigma_{i, j}= \pm 1\right)$ located on a square lattice which evolve in discrete time. At each time step, all spins $\sigma_{i, j}$ are updated according to the rule

$\sigma_{i, j}(t+1)= \begin{cases}\operatorname{sign}\left(\bar{\sigma}_{i, j+1}(t)+\bar{\sigma}_{i+1, j}(t)+\bar{\sigma}_{i, j}(t)\right) & \text { with probability } 1-p-q \\ +1 & \text { with probability } p \\ -1 & \text { with probability } q .\end{cases}$

For $p=q=0$ we have a deterministic evolution: each updated spin becomes equal to the majority of itself and of its northern and eastern neighbours. Non-zero $p$ and $q$ represent the effect of a noise which favours the + sign with probability $p$ and the sign with probability $q$. When $p=q$, the two orientations + and - play symmetric roles, whereas $p \not \neq q$ introduces a bias. It has been proved by Toom that for low-enough noise ( $p$ and $q$ sufficiently small, but otherwise unrestricted) two phases exist, in which the spins are predominantly +1 or -1 , respectively. We refer the reader to [8-11] for more discussion of the Toom model.

Using suitable initial or boundary conditions one can introduce a non-equilibrium interface between the two low-noise phases. It is such interfaces which are the object of study in this paper. Let us first produce the interface by initially filling some half space with the + phase and the complementary half space with the - phase. The directionality of the dynamics - information flows to the southwest-introduces interface motion at zero noise: an interface which makes an angle $\theta$ with the $x$-axis is stationary if $\theta \in[0, \pi / 2]$ and moves to the southwest if $\theta \in[\pi / 2, \pi]$; a maximum speed of $1 / \sqrt{2}$ is attained when $\theta=3 \pi / 4$. At low but non-zero noise strength, heuristic reasoning and computer simulations indicate that the interface remains flat on a macroscopic scale and attains a constant, orientation-dependent velocity (which will still be zero for some orientations). For example, the velocity of the interface at $\theta=3 \pi / 4$ decreases, while vertical and horizontal interfaces acquire a velocity directed to the west and south, respectively. (Interface velocity is discussed in more detail in section 7; see also figure 8.) On finer scales the interface fluctuates and broadens. The interface motion explains the stability of the low-noise phases [7]: whenever a droplet of the wrong phase is formed it is wiped out at constant speed by the interface travelling southwest and thus has no chance to stabilize.

Moving interfaces are intrinsically non-stationary; to maintain a static interface one must impose suitable boundary conditions. As already noted, in the Toom model with the NEC rule, dynamical information travels southwest. Thus, in order to enabie the boundaries to determine the bulk, we consider the system in the third quadrant only, and require the spins along the negative $x$-axis to be all +1 and along the negative $y$-axis to be all $-1: \sigma_{i, 0}=+1$ and $\sigma_{0, i}=-1$ for all $i<0$ and all $t$. With these boundary 
conditions the Toom model with non-zero noise establishes a unique steady state in the long time limit. When the noise level is low (but still non-zero), an interface separates the + phase in the upper portion of the quadrant from the - phase in the lower portion; since the interface must pass through the origin, we will refer to it as an anchored interface. Figure 1 shows typical steady-state configurations of the system for $p=q=0.02$ and $p=0.032, q=0.008$. The major objective of our undertaking here will be to investigate the interface fluctuations in this steady state, which appear to be much smaller than they would be in a similar equilibrium interface.

The remainder of the paper is organized as follows. In section 2 we introduce a low-noise approximation to the Toom interface. In spirit this is comparable to an sos approximation for equilibrium interfaces $[12,13]$. The dynamics of the interface slope is then described by an effective spin interchange dynamics in a $1 \mathrm{D}$ semi-infinite lattice, with the interface fluctuations corresponding to the steady-state fluctuations in the magnetization of the 1D system. In section 3 we present numerical results on the $1 \mathrm{D}$ system, discussing the mean and variance of the magnetization and the spin-spin correlations in the steady state. These correlations are negative and go to zero only very slowly, which explains the great suppression of fluctuations in the Toom interface as compared to the corresponding equilibrium one. The steady state of the spin exchange dynamics is characterized by certain stationarity relations. We discuss these in section 4 , and then use them in section 5 to derive an approximate scaling form for the spin-spin correlation function (the slope-slope correlation in the interface).

In section 6 we investigate an approximation of a different sort: we assume that the average value of the spin $S_{n+1}$ at site $n+1$, conditioned on certain information about the preceding spins $S_{1}, \ldots, S_{n}$, actually depends only on the magnetization $M_{n}=$ $\sum_{j=1}^{n} S_{j}$. The approximation leads to recursive relations for various expectations, which we iterate numerically for finite $n$. The large $n$ limit can be treated analytically in terms of certain nonlinear partial differential equations, which determine scaling exponents and functions in at least approximate agreement with the numerical results. Another approach is taken in section 7, where we apply the continuum theory of Kardar, Parisi and Zhang to Toom interfaces. The theory takes into explicit account the directional flow of information in the Toom model and seems to provide a fairly coherent picture of the interface fuctuations. Moreover, we encounter some novel features: the anchoring and the propagation of excitations along the interface convert the usual growth in time of the interface width to a broadening as a function of position in the steady

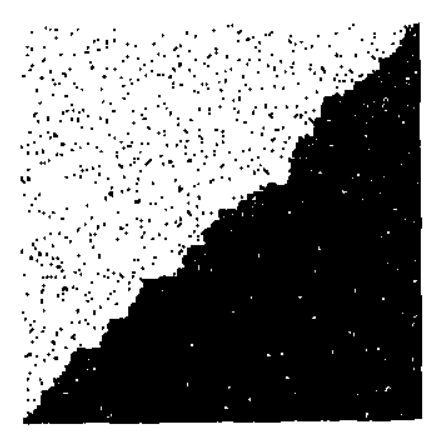

(a)

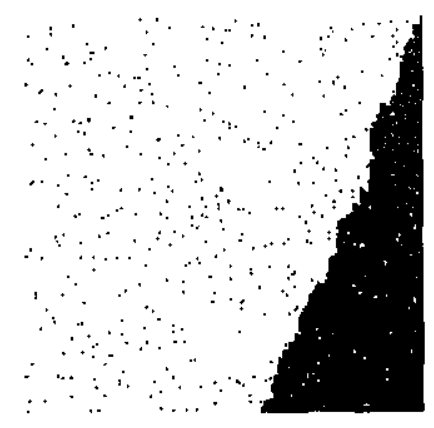

(b)

Figure 1. Typical steady-state system configurations for $(a) p=q=0.02$ and $(b) p=0.032$, $q=0.008$. 
state, and in the unbiased case $(p=q)$ the symmetry properties of the model change the exponent associated with this broadening. Finally, we give in section 8 some concluding remarks; three appendices contain additional material.

\section{The low-noise approximation}

At the zero-noise level $(p=q=0)$ the Toom model has a variety of time-independent configurations. In particular, we are interested in those defined by a single interface which is a directed path in the southwest direction; that is, the interface is a staircase, and all spins are - below it and + above it (cf figure 2 ).

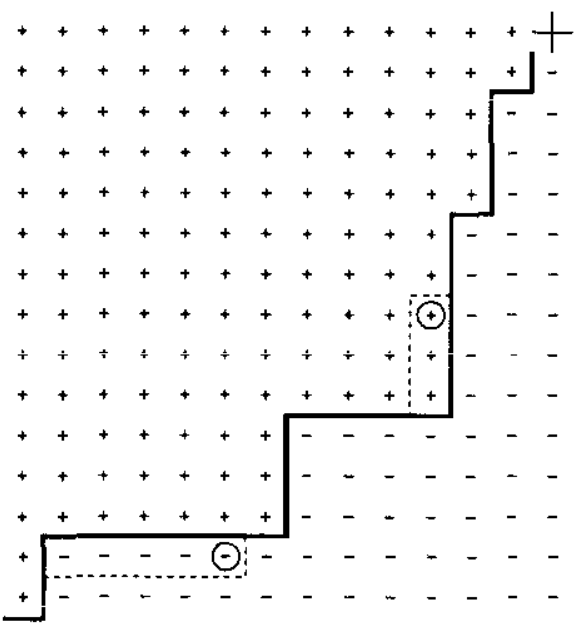

Figure 2. Staircase configuration of anchored interface in third quadrant. At very low noise, an error at one of the circled sites causes a transition to a new staircase indicated by the corresponding broken line.

In the presence of a very weak noise, small fluctuations are created. If one starts with a zero-noise configuration $\mathscr{C}_{0}$ of the staircase type considered above, then most of the fluctuations created by the noise (for example a - sign in a sea of + spins) have a very short lifetime: after such a fluctuation, the system returns quickly to its previous configuration $\mathscr{C}_{0}$. When the noise flips a spin $\sigma_{i, j}$ directly below a horizontal piece of the interface or directly to the left of a vertical piece, however, the system will jump rapidly to another configuration, as shown in figure 2 . Thus the problem in studying this interface is to understand how, under the effect of a weak noise, the interface fluctuates among its various zero-noise configurations.

Since the zero-noise interface is a directed walk, one can represent it (up to a uniform shift perpendicular to the interface) by a sequence of Ising spins $S_{n}= \pm 1$ : one takes the spin $S_{n}$ to be +1 if the $n$th link along the interface, southwest of the line $i+j=-1$, is vertical and to be -1 if this link is horizontal. Thus, each possible shape of the interface is represented by a sequence $S=\left\{\ldots, S_{-1}, S_{0}, S_{1}, S_{2}, \ldots\right\}$; our convention is that $n$ increases in a southwest direction along the interface. A transition of the type shown in figure 2 consists in flipping both the spin $S_{n}$ which corresponds to the link adjacent to the spin $\sigma_{i, j}$ and the first spin following $S_{n}$ which has a sign opposite to $S_{n}$. 
A little thought now shows, and a formal argument whose conclusions are summarized in appendix 2 confirms, that this dynamics of the interface slope can be represented in terms of a 1D model of Ising spins with the following (continuous time) spin exchange dynamics:

During an infinitesimal time interval $\mathrm{d} t$ each spin is updated independently. $\mathbf{A}+$ spin is exchanged with the first - spin to its right with probability $\lambda_{+} \mathrm{d} t$; similarly, a - spin is exchanged with the first + spin to its right with probability $\lambda_{-} \mathrm{d} t$. The ratio of these rates, $\lambda \equiv \lambda_{+} / \lambda_{-}$, corresponds to the limiting value of the ratio $q / p$ as the noise is reduced to zero.

More formally, the spin exchange rate $c(i, j)$ between two sites $i$ and $j$, with $i<j$, is given by

$c(i, j)=\lambda_{+} \frac{\left(1+S_{i}\right)}{2} \frac{\left(1-S_{j}\right)}{2} \prod_{i<k<j} \frac{\left(1+S_{k}\right)}{2}+\lambda_{-} \frac{\left(1-S_{i}\right)}{2} \frac{\left(1+S_{j}\right)}{2} \prod_{i<k<j} \frac{\left(1-S_{k}\right)}{2}$.

To study the anchored interface in the third quadrant, we use the same dynamics but restrict ourselves to the semi-infinite system $\left\{S_{n} \mid n=1,2,3, \ldots\right\}$; the spin $S_{1}$ now describes the orientation of a link whose north or east end is at $\left(-\frac{1}{2},-\frac{1}{2}\right)$. The resulting stationary state will be independent of initial conditions as long as the initial spin configuration contains infinitely many + and - spins (see the discussion of finite-size effects below). The height of the anchored interface relative to the $45^{\circ}$ diagonal corresponds in the $1 \mathrm{D}$ model, up to a factor $\sqrt{2}$, to the magnetization

$$
M_{n}=\sum_{k=1}^{n} S_{k}
$$

Clearly the quantities of interest are the steady-state fluctuations in the magnetization and the correlation functions of magnetization and spins.

The semi-infinite system has a very unusual property, refiecting the behaviour of the Toom model in the third quadrant: there are no finite-size effects. Specifically, if we restrict attention to the subsystem $1 \leqslant n \leqslant L$ and extend the dynamics so that a $+(-)$ spin with no spin of opposite sign to the right is simply flipped with probability $\lambda_{+} \mathrm{d} t\left(\lambda_{-} \mathrm{d} t\right)$ in time interval $\mathrm{d} t$, then the dynamics of the subsystem is identical to the dynamics induced from the infinite volume $(\boldsymbol{L}=\infty)$ system, as long as the infinite volüme system côntains añ infinite number of spins of both sigñs. In particulàr, to compute any correlation which involves only the spins $S_{1}, \ldots, S_{L}$, at equal or distinct times, we need consider only the subsystem $1 \leqslant n \leqslant L$. This permits in theory the exact calculation of any correlation function; for example, we find that in the steady state

$$
\left\langle S_{1}\right\rangle=\frac{1-\lambda}{1+\lambda} \quad\left\langle S_{1} S_{2}\right\rangle=1-\frac{12 \lambda}{(2 \lambda+1)(\lambda+2)} \cdots
$$

There are simple formulae for the probability that the first $n$ spins have the same sign:

$$
\left\langle\prod_{k=1}^{n}\left(\frac{1+S_{k}}{2}\right)\right\rangle=\prod_{k=1}^{n} \frac{1}{k \lambda+1} \quad\left\langle\prod_{k=1}^{n}\left(\frac{1-S_{k}}{2}\right)\right\rangle=\prod_{k=1}^{n} \frac{\lambda}{k+\lambda} .
$$

Expressions for other correlation functions quickly become complicated, and we see no way of obtaining asymptotic behaviour by this direct approach.

While very little more can be said with rigour about the steady state for the semi-infinite system, we can easily determine the steady state on a ring with periodic boundary conditions; in this case, a spin exchanges with the first spin of opposite sign in a counterclockwise direction. Consider a given spin configuration, in a ring of $N$ 
sites, with $N_{+}+$spins and $N_{-}=N-N_{+}-$spins, in which neither $N_{+}$nor $N_{-}$is zero. Then there are exactly $N_{+}\left(N_{-}\right)$exchanges occuring at rate $\lambda_{+}\left(\lambda_{-}\right)$which change this configuration, and one can easily check that there are the same number of exchanges at these rates which change other configurations to this one. Thus, for each $N_{+}, N_{-}$ there is a unique invariant measure in which all configurations with these numbers of + and - spins have equal weight. The thermodynamic limit $(N \rightarrow \infty)$ then yields invariant measures for the infinite system which are uniform product measures; any average magnetization $\mu=\left\langle S_{0}\right\rangle=\lim _{N \rightarrow \infty}\left(N_{+}-N_{-}\right) / N$, with $|\mu| \leqslant 1$, is allowed. Geometrically, the magnetization determines the slope of the corresponding (infinite) Toom interface: the angle $\alpha$ of the interface with the $x$-axis is given by

$$
\tan \alpha=\frac{1-\mu}{1+\mu} .
$$

It seems likely, but we have no proof, that these product measures are the only invariant measures of the doubly infinite system, and that the measure in the semi-infinite case is asymptotically a product measure far from the origin.

It follows that for a block of length $L$ in the doubly infinite system, or in such a block located far from the origin in the semi-infinite system, the magnetization has fluctuations of order $\sqrt{L}$. The naive 'equilibrium' guess would be that boundary conditions do not matter and that therefore also $\left\langle M_{n}^{2}\right\rangle \simeq n$ for the semi-infinite chain. In fact, as we will see, the fluctuations in this chain or equivalently in the anchored Toom interface at low noise grow much slower: there are strong anticorrelations due to the boundary. This is already evident from (2.4); for a product measure, the corresponding probability would decrease exponentially rather than factorially.

To understand the behaviour of the anchored interface it is useful first to calculate the current $J(\mu)$ produced by the spin dynamics, on the doubly infinite ID lattice, for a given value of the bias $\lambda$ and of the magnetization $\mu$. The current through some fixed bond of the lattice, say $(0,1)$, is determined by the fact that each spin exchange between sites on opposite sides of the bond contributes +2 to the current if the + spin is orginally to the left of the bond, and contributes -2 otherwise. Thus the average current through the bond is

$$
J(\mu)=\left\langle\sum_{i \leqslant 0, j \geqslant 1} c(i, j)\left(S_{i}-S_{j}\right)\right\rangle=\left\langle\sum_{n=1}^{\infty} n c(0, n)\left(S_{0}-S_{n}\right)\right\rangle .
$$

Using (2.1) and the fact that \langle\rangle is a product measure reduces (2.6) to a geometric series which sums to

$$
J(\mu)=2 \frac{\lambda_{+}(1+\mu)^{2}-\lambda_{-}(1-\mu)^{2}}{1-\mu^{2}} .
$$

Note that $J(\mu) \rightarrow \infty$ as $|\mu| \rightarrow 1$; this is the analogue of the divergence of the diffusion constant in a model with related dynamics, studied in [3] as an example of self-organized criticality. The normal velocity of the corresponding doubly infinite Toom interface is $J(\mu) / \sqrt{2\left(1+\mu^{2}\right)}$, so that for bias $\lambda$ the current vanishes at magnetization

$$
\mu=\frac{1-\sqrt{\lambda}}{1+\sqrt{\lambda}}
$$

For the semi-infinite system the current has to vanish; otherwise + or - spins would pile up somewhere. If we assume, as seems from simulations to be correct, that asymptotically the stationary state for this system is a product measure at some 
magnetization $\mu$, then (again asymptotically) the current must satisfy the condition $J(\mu)=0 ;(2.8)$ is indeed confirmed by simulations, as discussed in the next section. Thus, one expects that at low noise the anchored interface will asymptotically make an angle $\alpha$ with the $x$-axis determined by (2.8) and (2.5):

$$
\tan \alpha=\sqrt{\lambda}=\sqrt{\frac{q}{p}} .
$$

\section{Numerical results}

In this section we present the results of Monte Carlo simulations of the $1 \mathrm{D}$ spin model introduced in the previous section. We assume that $\lambda \leqslant 1$ for definiteness. The algorithm is then straightforward: for a chain of $L$ spins we choose at each step a spin $n \leqslant L$ at random. Then, with probability $\lambda$ if $S_{n}=+1$ and probability 1 if $S_{n}=-1$, we exchange it with the first spin of opposite sign which follows it or, if no such following spin exists between $n$ and $L$, we flip $S_{n}$ without any other change. We have already noted that this dynamics gives results for the distribution of spins $S_{1}, S_{2}, \ldots, S_{k}$ which are independent of $L$ as long as $L \geqslant k$, i.e. there are no finite-size effects.

As in other Monte Carlo simulations, we have to acknowledge, however, the fact that the successive configurations which are produced by the algorithm are not statistically independent. We may give a bound for the correlation time as follows. Consider two systems with distinct initial configurations subjected to the same dynamics, including noise, i.e. for which the spins are updated in the same order. The two systems will agree up to site $n+1$ (except for an overall sign) after the event in which spin 1 is updated, then spin 2 , then spin $3, \ldots$ continuing through spin $n$. Thus, they will agree completely, up to an overall sign, after Monte Carlo time $T_{0}$ with

$$
T_{0} \leqslant T \equiv \sum_{k=1}^{L} t_{k}
$$

where the times $t_{k}$ are independent identically distributed geometric random variables with $E\left(t_{k}\right)=L / \lambda$. If one defines a rescaled time $\tau$ by

$$
T=\left(L^{2} / \lambda\right) \tau
$$

then the density of $\tau$ becomes, for large $L$,

$$
P(\tau)=\sqrt{\frac{L}{2 \pi}} \exp -\frac{L(\tau-1)^{2}}{2} .
$$

Thus, after a time of order $L^{2}$, the system has certainly forgotten its initial state. Note that this time is on the simulation scale, which differs by a factor proportional to $L$ from the continuous time-scale introduced earlier.

We also computed $\left\langle M_{L}(0) M_{L}(t)\right\rangle$ directly in the simulation of the unbiased case and found that the effective decay time for this quantity grows as $L^{3 / 2}$. Somewhat surprisingly, this behaviour apparently does not correspond with that predicted by the second eigenvalue of the master equation which represents the dynamics (the maximum eigenvalue, associated with the steady state, is always unity). We computed this second eigenvalue for small systems in the unbiased case by a direct diagonalization; for all sizes $L \geqslant 5$ (we went up to 15 ) we found that the second eigenvalue in magnitude is $1-2 / L$. If this eigenvalue $1-2 / L$ (we can show that for all $L, 1-2 / L$ is an eigenvalue) remains always the second eigenvalue, this would imply that correlations must at very 
long times decay with correlation time proportional to $L$; nevertheless, the $L^{3 / 2}$ time appears to be the relevant one for our simulations.

Let us now come to the results of our simulations.

In table 1 , we give results for two values of the bias: $\lambda=1$ (no bias) and $\lambda=\frac{1}{4}$. The table includes values of $\left\langle M_{L}\right\rangle$ in the biased case $\left(\left\langle M_{L}\right\rangle=0\right.$ for $\left.\lambda=1\right)$, the truncated second moment $\left\langle M_{L}^{2}\right\rangle^{\mathrm{T}} \equiv\left\langle M_{L}^{2}\right\rangle-\left\langle M_{L}\right\rangle^{2}$, and the ratio $R_{L}$ defined by

$$
R_{L}=\frac{\left\langle\left(M_{L}-\left\langle M_{L}\right\rangle\right)^{4}\right\rangle}{\left(\left\langle M_{L}^{2}\right\rangle-\left\langle M_{L}\right\rangle^{2}\right)^{2}} .
$$

The simulation involved $4.6 \times 10^{6}$ updates per site in a system of $L=131072$ sites for $\lambda=1$, and $2.44 \times 10^{6}$ updates per site in a system of $L=16384$ sites for $\lambda=\frac{1}{4}$; error bars represent $95 \%$ confidence intervals obtained by subdividing long simulations into statistically independent pieces, using the forgetting time estimated above. The table includes also results of the collective variable approximation to be discussed in section 6 .

From these results it is clear that $\left\langle M_{L}^{2}\right\rangle-\left\langle M_{L}\right\rangle^{2}$ does not increase as $L$. Log-log plots of this quantity against $L$ are shown in figure 3 ; these suggest an increase as $L^{1 / 2}$ in the unbiased case, with a larger exponent in the case $\lambda=\frac{1}{4}$. (These plots show also the collective variable approximation of section 6 , which asymptotically grows as $L^{1 / 2}$ in the unbiased case and $L^{2 / 3}$ for any bias.) To determine the exponent more precisely we give in figure $4 \log -\log$ plots of $\left(\left\langle M_{L}^{2}\right\rangle-\left\langle M_{L}\right\rangle^{2}\right) / L^{1 / 2}$ against $L$ for $\lambda=1$ and of $\left(\left\langle M_{L}^{2}\right\rangle-\left\langle M_{L}\right\rangle^{2}\right) / L^{2 / 3}$ against $L$ for $\lambda=\frac{1}{4}$. (Again the collective variable results are included.) From the data, we see that for the range of values $2^{8} \leqslant L \leqslant L_{\max }$ (with

Table 1. Simulation results and collective variable approximation for the 1D spin model with system size $L$. In the unbiased case $(\lambda=1)$ we give the mean fluctuation $\left\langle M_{L}^{2}\right\rangle$ of the magnetization, from both simulation and $\mathrm{CV}$ approximation, the asymptotic $\mathrm{CV}$ prediction of $\sqrt{3 L / 2}$ for this fluctuation, and the ratio $R_{L}=\left\langle M_{L}^{4}\right\rangle /\left\langle M_{L}^{2}\right\rangle^{2}$ from simulation. In the biased case $\left(\lambda=\frac{1}{4}\right)$ we give the mean magnetization from the simulation, simulation and $C V$ values for the fluctuations (truncated expectations), and the value of $R_{L}$ (now calculated from truncated fluctuations).

\begin{tabular}{|c|c|c|c|c|c|c|c|c|}
\hline \multirow[b]{2}{*}{$L$} & \multicolumn{4}{|c|}{ Unbiased: $\lambda=1$} & \multicolumn{4}{|c|}{ Biased: $\lambda=\frac{1}{4}$} \\
\hline & $\left\langle M_{L}^{2}\right\rangle$ & $R_{L}$ & $\left\langle M_{L}^{2}\right\rangle_{C V}$ & $\sqrt{3 L / 2}$ & $\left\langle M_{L}\right\rangle$ & $\left\langle M_{L}^{2}\right\rangle^{T}$ & $R_{L}$ & $\left\langle M_{L}^{2}\right\rangle_{\mathrm{CV}}^{\mathrm{T}}$ \\
\hline 2 & 1.33 & 3.00 & 1.33 & 1.73 & -1.02 & $1.18 \pm 0.01$ & 2.04 & 1.18 \\
\hline 4 & 1.92 & 2.95 & 1.92 & 2.45 & -1.77 & $1.72 \pm 0.01$ & 2.99 & 1.73 \\
\hline 8 & 2.73 & 3.00 & 2.76 & 3.46 & -3.22 & $2.51 \pm 0.01$ & 3.00 & 2.54 \\
\hline 16 & 3.89 & 3.02 & 4.02 & 4.90 & -6.02 & $3.64 \pm 0.01$ & 3.04 & 3.74 \\
\hline 32 & $5.57 \pm 0.02$ & 3.03 & 5.88 & 6.92 & -11.5 & $5.29 \pm 0.03$ & 3.05 & 5.54 \\
\hline 64 & $8.03 \pm 0.03$ & 3.04 & 8.60 & 9.80 & -22.4 & $7.73 \pm 0.04$ & 3.07 & 8.21 \\
\hline 128 & $11.58 \pm 0.04$ & 3.05 & 12.53 & 13.86 & -44.1 & $11.34 \pm 0.06$ & 3.07 & 12.16 \\
\hline 256 & $16.7 \pm 0.1$ & 3.04 & 18.16 & 19.60 & -87.2 & $16.75 \pm 0.1$ & 3.08 & 17.98 \\
\hline 512 & $24.2 \pm 0.1$ & 3.04 & 26.19 & 27.71 & -173.1 & $24.7 \pm 0.2$ & 3.06 & 26.59 \\
\hline 1024 & $34.9 \pm 0.3$ & 3.05 & 37.60 & 39.19 & -344.6 & $36.8 \pm 0.3$ & 3.07 & 39.43 \\
\hline 2048 & $50.6 \pm 0.4$ & 3.03 & 53.78 & 55.43 & -687.0 & $55.6 \pm 0.7$ & 3.07 & 58.75 \\
\hline 4096 & $72.7 \pm 1.2$ & 3.04 & 76.69 & 78.38 & -1371 & $83.9 \pm 1.1$ & 3.06 & 88.10 \\
\hline 8192 & $106 \pm 2.5$ & 3.05 & 109.1 & 110.9 & -2738 & $127 \pm 2.5$ & & 133.2 \\
\hline 16384 & $151 \pm 3$ & 3.02 & 155.0 & 156.8 & -5471 & $192 \pm 3.5$ & & 203.0 \\
\hline 65536 & $313 \pm 4$ & & 311.7 & 313.5 & & & & 482.5 \\
\hline 131072 & $451 \pm 7$ & & 441.6 & 443.4 & & & & 750.9 \\
\hline 262144 & & & 625.3 & 627.1 & & & & 1174.1 \\
\hline
\end{tabular}



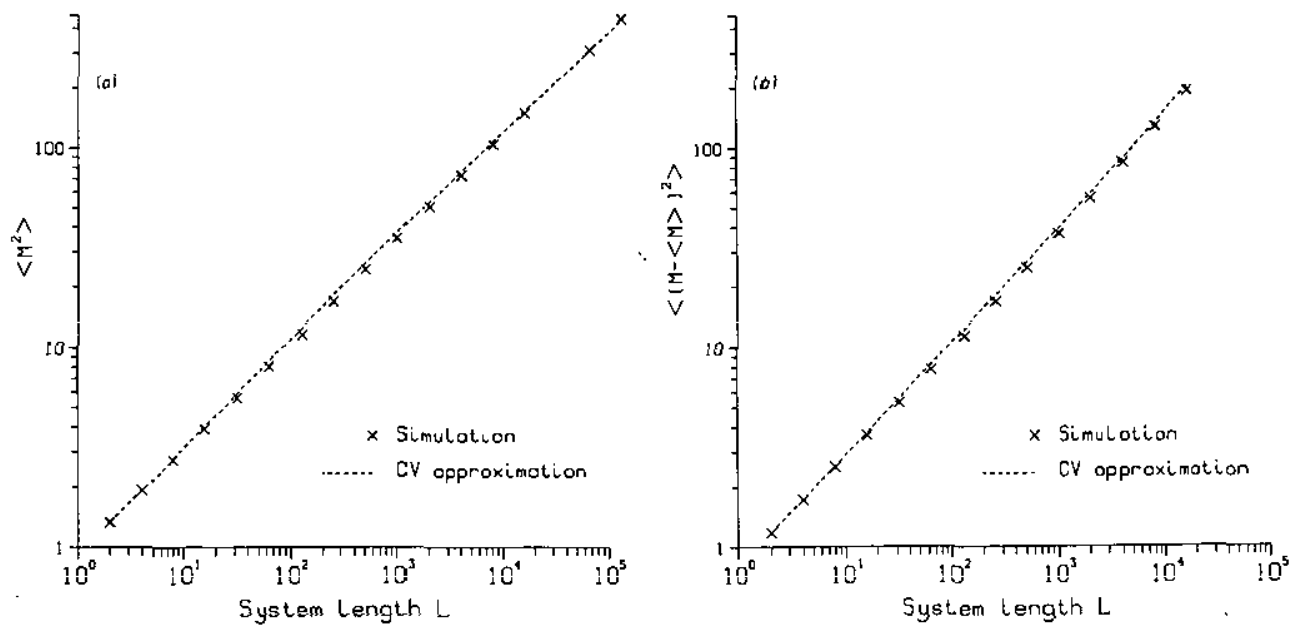

Figure 3. Mean square fluctuation in magnetization against system size: $(a)$ unbiased case, $\lambda=1 ;(b)$ biased case, $\lambda=\frac{1}{4}$.
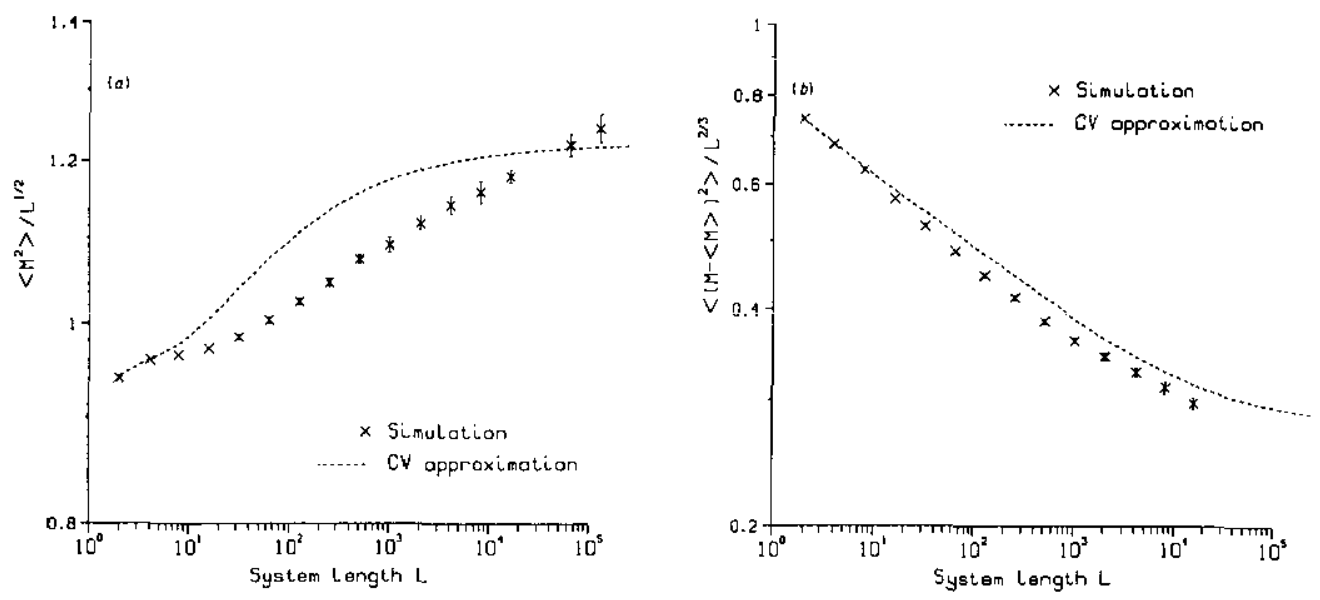

Figure 4. Mean square fluctuation in magnetization, normalized by exponent of $\mathrm{CV}$ or KPZ prediction, against system size $L:(a)$ unbiased case, $\lambda=1$, with normalization by $L^{-1 / 2}$; (b) biased case, $\lambda=\frac{1}{4}$, with normalization by $L^{-2 / 3}$.

$L_{\max }=2^{17}$ for $\lambda=1, L_{\max }=2^{14}$ for $\lambda=\frac{1}{4}$ ) our results are consistent with a power law,

$$
\left\langle M_{L}^{2}\right\rangle-\left\langle M_{L}\right\rangle^{2}=L^{2 \nu}
$$

with

$$
\nu \simeq \begin{cases}0.265 & \text { if } \lambda=1 \\ 0.285 & \text { if } \lambda=\frac{1}{4} .\end{cases}
$$

Clearly the fluctuations of $M_{L}$ are much smaller than expected from the calculation of section 2 for the ring geometry, which would give $\nu=0.5$; again, this is due to antiferromagnetic correlations, which we discuss further below. In the next sections we shall discuss several approximate treatments which explain, at least partially, these 
anomalously small fluctuations. These approximate models suggest that in fact (3.5) holds asymptotically with

$$
\nu= \begin{cases}\frac{1}{4} & \text { if } \lambda=1 \\ \frac{1}{3} & \text { if } \lambda \neq 1 .\end{cases}
$$

We measured several other quantities in our simulations. Referring again to table 1 , we see that, in the unbiased case, $R_{L}$ is rather close to 3 over the range of sizes considered. A more detailed study of the shape of the distribution of $M_{L}$ in this case shows that the distribution is indeed close to Gaussian. From our data, however, it is hard to decide whether the difference between $R_{L}$ and 3 is going to vanish for $L \rightarrow \infty$ or not. In the biased case, $R_{L}$ is somewhat further from 3 ; in fact, the collective variable approximation predicts a non-Gaussian distribution in the biased case.

We measured also the average magnetization $\left\langle M_{L}\right\rangle$ at several values of the bias. To a good approximation, $\left\langle M_{L}\right\rangle$ increases linearly with $L$ and the ratio $\mu=\left\langle M_{L}\right\rangle / L$ is given by the expression (2.8) (see figure 5 for a plot of $\left\langle M_{L}\right\rangle / L$ against the bias $\lambda$ ).

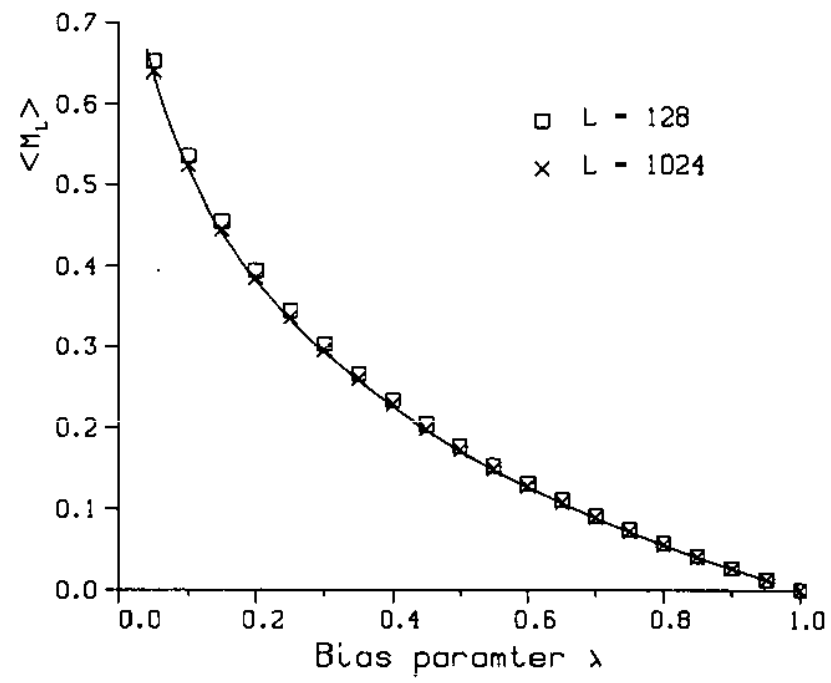

Figure 5. Mean magnetization against bias parameter $\lambda$. Simulation at system sizes $L=128$ and $L=1024$ compared with theoretical prediction (2.8).

The last quantity that we measured with some accuracy is the correlation function $\left\langle S_{i} S_{j}\right\rangle .\left\langle S_{i} S_{j}\right\rangle$ is negative for all $i \neq j$ and decreases in magnitude as $i, j \rightarrow \infty$ even for $|i-j|$ fixed; that is, the state does asymptotically behave as a product measure. The approach is very slow, however, going in general as the square root of the distance from the origin. Indeed, as $i$ and $j$ become large, $\left\langle S_{i} S_{j}\right\rangle$ seems to approach a scaling form

$$
\left\langle S_{i} S_{j}\right\rangle \simeq i^{-1 / 2} F\left(\frac{j-i}{i^{1 / 2}}\right)
$$

as shown in figures 6 and 7 , where we have plotted the scaled correlation $-i^{1 / 2}\left\langle S_{i} S_{j}\right\rangle$ as a function of $(j-i) / i^{1 / 2}$, in the unbiased case, for several fixed values of $i$. (Figure 7 also contains a theoretically derived curve, to be discussed in section 5.) Whether the scaling form (3.8) becomes true in the limit $L \rightarrow \infty$ or whether it is only approximately valid for the sizes $L$ we have considered here is again an open question. 


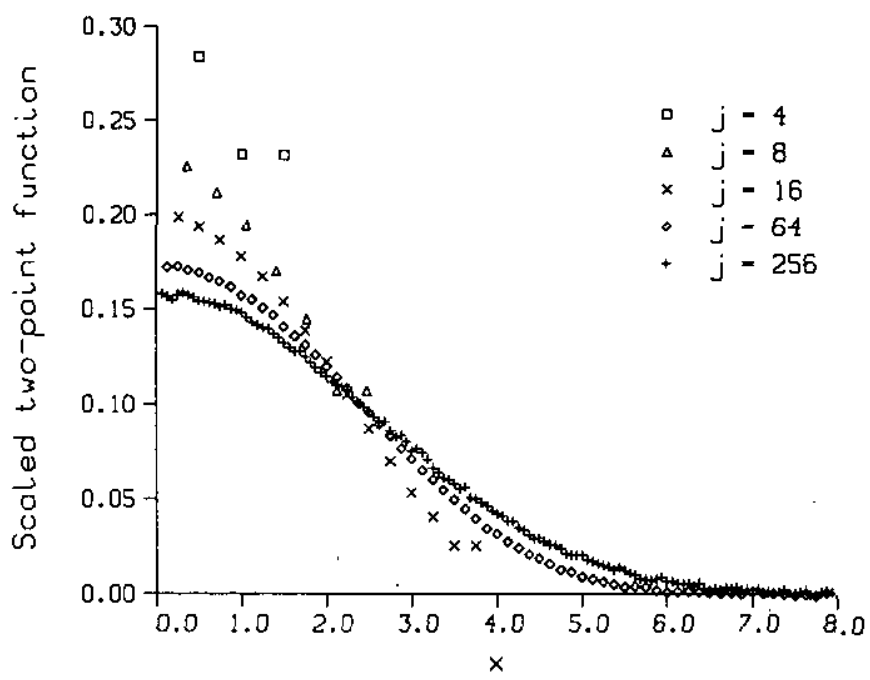

Figure 6. Two-point correlation function: results of simulation for various system sizes.

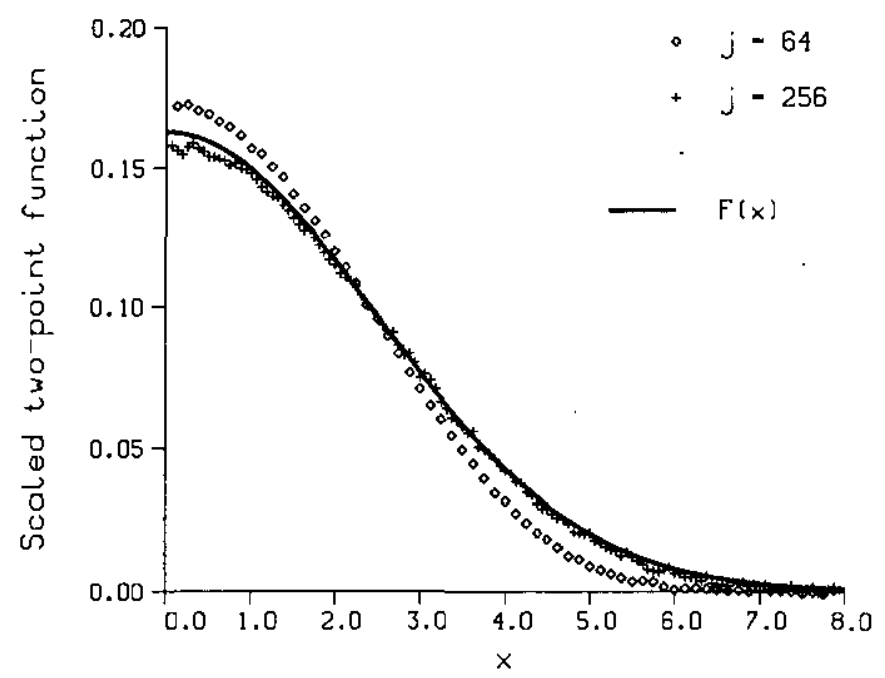

Figure 7. Two-point correlation function: results of simulation for $L=64$ and $L=256$ compared with theoretical prediction (5.8).

In summary: in the unbiased case, the main effect observed in our simulations is that the fluctuations of $M_{L}$ are of order approximately $L^{1 / 4}$ instead of $L^{1 / 2}$. Both the distribution of $M_{L}$ and the correlation functions appear to be rather simple: the distribution of $M_{L}$ seems to be Gaussian whereas the correlations seem to satisfy the scaling form (3.8). However, within the accuracy of our numerical data, it seems that if, in fact, $\nu=\frac{1}{4}, R_{L} \rightarrow 3$ and the correlation function scales in the limit $L \rightarrow \infty$, then the convergence to this asymptotic regime is extremely slow; thus, it is possible that some or all of these conclusions do not hold. Conclusions in the biased case are even less clear; certainly our simulations give at best weak support for the 'theoretical' value $\nu=\frac{1}{3}$. 
Of course, it is possible that our numerical data suffer from some small systematic error (i.e. a bad random number generator). In all the tests we did, however, our results remained unchanged when we used different algorithms for generating random numbers.

\section{Steady-state relations}

For the dynamics of the model defined in section 2 , it is possible to write many stationarity conditions which express the fact that the system is in its steady state. We are going to derive a few of these relations which we shall use later on to develop several kinds of approximations.

The main idea to obtain a stationarity condition is to choose some function $\Psi(S)$ of the spin configuration $S=\left\{S_{1}, \ldots, S_{L}\right\}$ and to write that the expectation $\langle\Psi\rangle$ remains unchanged under the dynamics;

$$
\frac{\mathrm{d}}{\mathrm{d} t}\langle\Psi\rangle=0
$$

More explicitly, (4.1) can be expressed as

$$
\left\langle\sum_{i<j} c(i, j)\left(\Psi\left(S^{i j}\right)-\Psi(S)\right)\right\rangle=0
$$

Here $c(i, j)$ is the spin exchange rate $(2.1)$ and $S^{i j}$ denotes the spin configuration $S$ with the spins at sites $i$ and $j$ interchanged.

For simplicity, let us first consider the unbiased case $\lambda=1$. Let $K_{i}$ be defined as the number of successive spins directly preceding $S_{i}$ (including $S_{i}$ itself) which have the same orientation as $S_{i}$. Clearly $K_{i}$ satisfies

$$
K_{1}=1 \quad K_{i+1}=1+\frac{1+S_{i} S_{i+1}}{2} K_{i}
$$

from which we may derive

$$
K_{i}=2+\sum_{i>p>q \geqslant 0} \frac{S_{i-p} S_{i-q}}{2^{p-1}}+\sum_{i>p>q>r>s \geqslant 0} \frac{S_{i-p} S_{i-q} S_{i-r} S_{i-s}}{2^{p-1}}+\ldots
$$

Having introduced $K_{i}$, we can now obtain some stationarity relations.

We first take $\Psi$ to be an arbitrary function $\Phi$ of the magnetization $M_{n}$. Then, during an infinitesimal time interval $\mathrm{d} t, M_{n}$ remains unchanged with probability $1-K_{n} \mathrm{~d} t$ and $M_{n}$ becomes $M_{n}-2 S_{n}$ with probability $K_{n} \mathrm{~d} t$. Therefore the stationarity relation (4.1) becomes

$$
\left\langle K_{n} \Phi\left(M_{n}\right)\right\rangle=\left\langle K_{n} \Phi\left(M_{n}-2 S_{n}\right)\right\rangle
$$

or, equivalently,

$$
\left\langle K_{n}\left(\Phi\left(M_{n-1}+S_{n}\right)-\Phi\left(M_{n-1}-S_{n}\right)\right)\right\rangle=0 .
$$

Since $\Phi(M)$ can be anything, one can choose $\Phi(M)=M^{2}$. Then (4.5), (4.6) become

$$
\left\langle K_{n} M_{n} S_{n}\right\rangle=\left\langle K_{n}\right\rangle \quad \text { or } \quad\left\langle K_{n} S_{n} M_{n-1}\right\rangle=0
$$


Relations (4.5)-(4.7) are valid for any $n \geqslant 1$; (4.7) should be compared with the corresponding result for a product measure with $\left\langle S_{i}\right\rangle=0$, for which $\left\langle K_{n} S_{n} M_{n-1}\right\rangle \rightarrow 2$ as $n \rightarrow \infty$. In fact, one can notice that a consequence of $(4.6)$ is that

$$
\left\langle K_{n} S_{n} \phi\left(M_{n-1}\right)\right\rangle=0
$$

for all functions $\phi$, so that the random variable $K_{n} S_{n}$, which is the signed size of the block to the left of $n$, has zero expectation for every value of the magnetization $M_{n-1}$.

One can obtain other stationarity relations by considering other functions $\Psi(S)$, for example

$$
\Psi(S)=S_{i} S_{j}
$$

where we take $i<j$. In this case we first remark that the spins $S_{i}$ and $S_{j}$ can exchange with each other only if $S_{i}=S_{i+1}=\ldots=S_{j-1} \neq S_{j}$, that is, if $X_{i j} \equiv \Pi_{i \leq k<j}\left(1-S_{k} S_{j}\right) / 2=1$; in the time interval $\mathrm{d} t$ the probability of this exchange is thus $X_{i j} \mathrm{~d} t$. Now we note that during this time interval the pair $\left(S_{i}, S_{j}\right)$ becomes $\left(-S_{i}, S_{j}\right)$ if either $S_{i}$ exchanges with a spin $S_{k}$ with $k>i$ but $k \neq j$, an event which by the above remark has probability $\left(1-X_{i j}\right) \mathrm{d} t$, or if some spin $S_{k}$ with $k<i$ exchanges with $S_{i}$, an event with probability $\left[K_{i-1}\left(1-S_{i} S_{i-1}\right) / 2 \mathrm{~d} t\right.$. Similarly, $\left(S_{i}, S_{j}\right)$ becomes $\left(S_{i},-S_{j}\right)$ with probability $1 \cdot \mathrm{d} t+$ $\left[K_{j-1}\left(1-S_{j} S_{j-1}\right) / 2-X_{i j}\right] \mathrm{d} t$. Since in each case the change in $\Psi$ is $\mathrm{d} \Psi=-2 S_{i} S_{j}$, setting $\langle\mathrm{d} \Psi\rangle$ to zero yields

$4\left\langle S_{i} S_{j}\right\rangle+\left\langle K_{i-1} S_{i} S_{j}\right\rangle-\left\langle K_{i-1} S_{i-1} S_{j}\right\rangle+\left\langle K_{j-1} S_{i} S_{j}\right\rangle-\left\langle K_{j-1} S_{i} S_{j-1}\right\rangle=-4\left\langle X_{i j}\right\rangle$

which can be rewritten using (4.3) as

$$
\left\langle K_{i} S_{i} S_{j}\right\rangle-\left\langle K_{i-1} S_{i-1} S_{j}\right\rangle+\left\langle K_{j} S_{i} S_{j}\right\rangle-\left\langle K_{j-1} S_{i} S_{j-1}\right\rangle=-2\left\langle\prod_{i \leqslant k<j} \frac{1-S_{j} S_{k}}{2}\right\rangle .
$$

When $i$ and $j$ are far apart the right-hand side of (4.11) can be neglected (because events for which $X_{i j}$ is not zero are rare). Thus, for $|i-j| \gg 1$ we have approximately

$$
\left\langle K_{i} S_{i} S_{j}\right\rangle-\left\langle K_{i-1} S_{i-1} S_{j}\right\rangle+\left\langle K_{j} S_{i} S_{j}\right\rangle-\left\langle K_{j-1} S_{i} S_{j-1}\right\rangle=0 .
$$

One can generalize (4.11) or (4.12) to an arbitrary number of spins. For example, if the sites $i, j, k, l$ are all distant from each other and far from the beginning of the chain, one gets

$$
\begin{aligned}
\left\langle K_{i} S_{i} S_{j} S_{k} S_{l}\right\rangle- & \left\langle K_{i-1} S_{i-1} S_{j} S_{k} S_{l}\right\rangle+\left\langle K_{j} S_{i} S_{j} S_{k} S_{l}\right\rangle-\left\langle K_{j-1} S_{i} S_{j-1} S_{k} S_{l}\right\rangle \\
& +\left\langle K_{k} S_{i} S_{j} S_{k} S_{l}\right\rangle-\left\langle K_{k-1} S_{i} S_{j} S_{k-1} S_{l}\right\rangle+\left\langle K_{l} S_{i} S_{j} S_{k} S_{l}\right\rangle-\left\langle K_{l-1} S_{i} S_{j} S_{k} S_{l-1}\right\rangle=0
\end{aligned}
$$

One can obtain many other stationarity relations by writing that other expectations $\langle\Psi\rangle$ are stationary. The main problem with these relations is that they all involve $K_{n}$ and hence that, when (4.4) is used, they generate a hierarchy of relations between pair, four-point, six-point, and higher-order correlation functions, from which it is hard to extract closed expressions for the pair correlations. In the next sections we will develop closure approximations for this hierarchy.

Before doing so, let us give a stationarity relation valid in the biased case $(\lambda \leqslant 1)$. By writing that an arbitrary function $\Phi(M)$ is stationary, one gets

$$
\lambda_{+}\left(\left\langle K_{n}^{(+)} \Phi\left(M_{n}\right)\right\rangle-\left\langle K_{n}^{(+)} \Phi\left(M_{n}-2\right)\right\rangle\right)=\lambda_{-}\left(\left\langle K_{n}^{(-)} \Phi\left(M_{n}+2\right)\right\rangle-\left\langle K_{n}^{(-)} \Phi\left(M_{n}\right)\right\rangle\right)
$$


where $K_{i}^{(+)}\left(K_{i}^{(-)}\right.$respectively) is the number of consecutive + spins (respectively - ) preceeding spin $i$, including $i$ itself. The reasoning used to derive this relation is a direct generalization of that leading to (4.5). Stationarity relations for correlation functions, generalizing (4.11) and (4.13), may of course be derived similarly.

Equation (4.14) can be rewritten as follows. Let $W_{n}(m)$ denote the probability that $M_{n}=m$, and set

$$
U_{n}^{( \pm)}(m)=\lambda_{ \pm}\left\langle K_{n}^{( \pm)} \mid M_{n}=m \pm 1\right\rangle W_{n}(m \pm 1)
$$

with $\langle\mid\rangle$ denoting conditional expectation, so that (4.14) becomes

$\sum_{m}\left(U_{n}^{(+)}(m-1)-U_{n}^{(-)}(m-1)\right) \Phi(m)=\sum_{m}\left(U_{n}^{(+)}(m+1)-U_{n}^{(-)}(m+1)\right) \Phi(m)$.

Since $\Phi$ is arbitrary, we must have

$$
U_{n}^{(+)}(m-1)-U_{n}^{(-)}(m-1)=U_{n}^{(+)}(m+1)-U_{n}^{(-)}(m+1)
$$

for all integers $m$; since $M_{n} \leqslant n$ and hence $U_{n}^{( \pm)}(m)=0$ for $m$ sufficiently large, (4.17) implies that

$$
U_{n}^{(+)}(m)=U_{n}^{(-)}(m)
$$

for all $m$. Equation (4.18) will play a key role in section 6 .

\section{The scaling form of the pair correlation function}

From the stationarity relations obtained in section 4 one may obtain, by making two assumptions, an analytic expression for the correlation function $\left\langle S_{i} S_{j}\right\rangle$. We will limit ourselves to the unbiased case $(\lambda=1)$.

First, we assume that, in the asymptotic regime, the pair correlations are much larger than four-point correlations and any higher correlation functions. Then, by neglecting the higher correlations, we may replace the stationarity relation (4.12) by a relation for the pair correlation only. To do so we insert the expression (4.4) for $\boldsymbol{K}_{i}$ into (4.12) and discard all correlation functions higher than pair correlations; we obtain

$$
\sum_{p \geqq 0} 2^{-p}\left(\left\langle S_{i-p} S_{j}\right\rangle+\left\langle S_{i} S_{j-p}\right\rangle-\left\langle S_{i-1-p} S_{j}\right\rangle-\left\langle S_{i} S_{j-1-p}\right\rangle\right)=0
$$

for $i \gg 1, j \gg 1$ and $|i-j| \gg 1$. Second, we assume that in this regime $\left\langle S_{i} S_{j}\right\rangle$ has the scaling form (3.8)

$$
\left\langle S_{i} S_{j}\right\rangle=\frac{1}{i^{1 / 2}} F\left(\frac{j-i}{i^{1 / 2}}\right) .
$$

For large $i$ and $j$, and writing $x=(j-i) / i^{1 / 2}$, we then have

$$
\begin{aligned}
& \left\langle S_{i} S_{j-p}\right\rangle=\frac{1}{i^{1 / 2}} F(x)-\frac{p}{i} F^{\prime}(x)+\frac{p^{2}}{2 i^{3 / 2}} F^{\prime \prime}(x)+\mathrm{O}\left(i^{-2}\right) \\
& \left\langle S_{i-p} S_{j}\right\rangle=\frac{1}{i^{1 / 2}} F(x)+\frac{p}{i} F^{\prime}(x)+\frac{p}{2 i^{3 / 2}}\left(F(x)+x F^{\prime}(x)+p F^{\prime \prime}(x)\right)+\mathrm{O}\left(i^{-2}\right) .
\end{aligned}
$$

By substituting (5.3) and (5.4) into (5.1) we obtain for the leading non-vanishing order

$$
F(x)+x F^{\prime}(x)+6 F^{\prime \prime}(x)=0 .
$$


The general solution of $(5.5)$ is

$$
F(x)=A \exp \left(-\frac{x^{2}}{12}\right)+B \exp \left(-\frac{x^{2}}{12}\right) \int_{0}^{x} \exp \left(\frac{t^{2}}{12}\right) \mathrm{d} t
$$

If we assume, as suggested by simulations, that the correlation function decays faster than $1 / x$ for large $x$ and that $\left\langle M_{n}^{2}\right\rangle$ does not increase linearly with $n$, then we must have

$$
B=0 \quad \int_{0}^{\infty} F(x) \mathrm{d} x=-\frac{1}{2}
$$

and therefore

$$
F(x)=-\frac{1}{\sqrt{12 \pi}} \exp \left(-\frac{x^{2}}{12}\right)
$$

This expression for the scaling form of $\left\langle S_{i} S_{j}\right\rangle$ is plotted in figure 7 , together with the results of the numerical simulation. We see that the agreement with the data for the largest size $(j=256, i<j)$ looks excellent. However, figures 6 and 7 suggest that the scaled correlation function at that size has not yet reached its large $n$ limit, so it is possible that the result (5.8) of the above apprxoimation is not the true scaling function.

One can try to extend this approach to calculate other quantities like $\left\langle M_{n}^{2}\right\rangle$, higher moments of $M_{n}$, or the correlation functions in the biased case. In all these cases, one has to make some additional assumptions; for example, to calculate $\left\langle M_{n}^{2}\right\rangle$ for large $n$, one needs to know the leading correction to the scaling form (5.2), or alternatively the four-point correlation (see appendix 1). In the biased case, odd correlation functions are also non-zero, making the above assumptions even more delicate.

In any case, we have not succeeded, so far, in extending the above assumptions in a way which would satisfy all the stationarity relations that we have and which would give a good agreement with our present numerical data. However, it is reasonable to think that, if some scaling holds for large $L$, then by introducing the right scaling forms for the correlation functions in the stationarity relations, one could get the exact analytic expressions for the asymptotic properties of the magnetization.

\section{A collective variable approximation}

In this section we introduce an approximate treatment of the stationarity condition (4.18) which leads to an (approximate) recursive scheme for calculating the distribution of the total magnetization in our model. One way to view this approximation is as the construction of an approximate measure which satisfies some of the stationarity conditions obtained in section 4 , specifically (4.5) and (4.6) in the unbiased case or more generally (4.14).

Our starting point is the recursive equation satisfied by the probability $W_{n}(m)$ that $M_{n}=m$, which, with the definition

$$
\left\langle S_{n+1} \mid M_{n}=m\right\rangle \equiv H_{n}(m)
$$

becomes

$$
W_{n+1}(m)=\frac{1}{2}\left(1+H_{n}(m-1)\right) W_{n}(m-1)+\frac{1}{2}\left(1-H_{n}(m+1)\right) W_{n}(m+1)
$$


and (4.15), which we write in the form

$$
\begin{aligned}
U_{n+1}^{( \pm)}(m)= & \lambda_{ \pm} \sum_{k=1}^{\infty} k \operatorname{Prob}\left\{K_{n+1}^{( \pm)}=k, M_{n+1}=m \pm 1\right\} \\
= & \lambda_{ \pm} \operatorname{Prob}\left\{S_{n+1}= \pm 1 \mid M_{n}=m\right\} W_{n}(m)+\lambda_{ \pm} \sum_{k=1}^{\infty} k \operatorname{Prob}\left\{K_{n}^{( \pm)}=k, M_{n}=m\right\} \\
& \times \operatorname{Prob}\left\{S_{n+1}= \pm 1 \mid K_{n}^{( \pm)}=k, M_{n}=m\right\} .
\end{aligned}
$$

We now define approximations $\hat{U}^{( \pm)}, \hat{W}$ and $\hat{H}$ of $U^{( \pm)}, W$ and $H$ by retaining the recurrence (6.2),

$\hat{W}_{n+1}(m)=\frac{1}{2}\left(1+\hat{H}_{n}(m-1)\right) \hat{W}_{n}(m-1)+\frac{1}{2}\left(1-\hat{H}_{n}(m+1)\right) \hat{W}_{n}(m+1)$

and the equality (4.18),

$$
\hat{U}_{n}^{(+)}(m)=\hat{U}_{n}^{(-)}(m)
$$

and making the collective variable approximation

$$
\left\langle S_{n+1} \mid K_{n}^{( \pm)}=k, M_{n}=m\right\rangle \simeq\left\langle S_{n+1} \mid M_{n}=m\right\rangle
$$

in (6.3), to obtain

$$
\hat{U}_{n+1}^{( \pm)}(m)=\frac{1}{2}\left(1 \pm \hat{H}_{n}(m)\right)\left(\lambda_{ \pm} \hat{W}_{n}(m)+\hat{U}_{n}^{( \pm)}(m \mp 1)\right) .
$$

Let us denote the common value (6.5) by $\hat{U}_{n}$. Equating the results of the evolution (6.7) of $\hat{U}^{(+)}$and $\hat{U}^{(-)}$yields

$$
\begin{aligned}
\hat{U}_{n+1}(m) & =\frac{1}{2}\left(1-\hat{H}_{n}(m)\right)\left(\lambda_{-} \hat{W}_{n}(m)+\hat{U}_{n}(m+1)\right) \\
& =\frac{1}{2}\left(1+\hat{H}_{n}(m)\right)\left(\lambda_{+} \hat{W}_{n}(m)+\hat{U}_{n}(m-1)\right) .
\end{aligned}
$$

Equation (6.8) can be solved for $\hat{H}_{n}$ :

$$
\hat{H}_{n}(m)=\frac{\hat{U}_{n}(m+1)-\hat{U}_{n}(m-1)+\left(\lambda_{-}-\lambda_{+}\right) \hat{W}_{n}(m)}{\hat{U}_{n}(m+1)+\left(\lambda_{-}+\lambda_{+}\right) \hat{W}_{n}(m)+\hat{U}_{n}(m-1)} .
$$

Then

$$
\hat{U}_{n+1}(m)=\frac{\left(\lambda_{-} \hat{W}_{n}(m)+\hat{U}_{n}(m+1)\right)\left(\lambda_{+} \hat{W}_{n}(m)+\hat{U}_{n}(m-1)\right)}{\hat{U}_{n}(m+1)+\left(\lambda_{-}+\lambda_{+}\right) \hat{W}_{n}(m)+\hat{U}_{n}(m-1)} .
$$

Equations (6.9), (6.10) and (6.4) determine $\hat{H}, \hat{U}$ and $\hat{W}$ recursively.

From these recursions we may approximate $\left\langle M_{n}^{2}\right\rangle$ :

$$
\left\langle M_{n}^{2}\right\rangle \simeq \sum_{m} m^{2} \hat{W}_{n}(m)
$$

This approximation is compared in figures 3 and 4 with the results of the Monte Carlo simulations discussed in section 3 . We see that the agreement is rather good, particularly in the unbiased case. Figure 4 suggests (and the theoretical discussion below confirms) that the asymptotic value of the exponent $\nu$ (see (3.5)) in the collective variable approximation is given by (3.7): $\frac{1}{4}$ for the unbiased case, $\frac{1}{3}$ for the biased case. The fact that the effective exponent for smaller values of $n$ varies from these asymptotic values in the direction of the effective exponent (3.6) obtained in simulating the true spin exchange model suggests that the asymptotic regime may not have been reached in the numerical calculations of section 3 . 
There is an alternate way to view these recurrence relations. We may in fact replace (6.6) by the stronger assumption that the distribution of $S_{n+1}$, conditioned on all information about previous spins, depends only on the collective variable $M_{n}$ :

$$
\left\langle S_{n+1} \mid S_{1}, \ldots, S_{n}\right\rangle=\hat{H}_{n}\left(M_{n}\right)
$$

where as usual $M_{n}=\sum_{i=1}^{n} S_{i}$. Once $\hat{H}$ is specified, (6.12) is sufficient to determine completely a measure on all spin configurations, which we term the collective variable (cv) measure. Now $\hat{W}_{n}$ is the actual probability distribution of $M_{n}$ in the cv measure, and

$$
\hat{U}_{n}^{( \pm)}(m)=\lambda_{ \pm}\left\langle K_{n}^{( \pm)} \mid M_{n}=m \pm 1\right\rangle_{\mathrm{CV}} \hat{W}_{n}(m \pm 1)
$$

where \langle\rangle$_{\mathrm{CV}}$ denotes expectations in the CV measure. Imposition of the condition (6.5) now determines $\hat{H}$, and all the recurrence relations above follow. The consequences we have derived from the assumption (6.6) may thus be regarded as referring to actual expectation values in the $\mathrm{CV}$ measure; in particular, the second moment defined in (6.11) is in fact just $\left\langle M_{n}^{2}\right\rangle_{\mathrm{CV}}$.

Condition (6.5) may be regarded as a requirement that the probability distribution of the magnetization $M_{n}$ in the cV measure be instantaneously stationary under the dynamics of section 2; that is, if we let \langle\rangle$^{t}$ denote the expectation at time $t$ under that dynamics, with initial distribution given by \langle\rangle$^{0}=\langle\rangle_{\mathrm{CV}}$, then for any function $\Phi$

$$
\left.\frac{\mathrm{d}}{\mathrm{d} t}\left\langle\Phi\left(M_{n}\right)\right)^{t}\right|_{t=0}=0
$$

Expectation values of quantities other than functions of the total magnetization may of course also be calculated in the $\mathrm{CV}$ measure, and compared with simulations. We consider here the two-point correlation function $\left\langle S_{i} S_{j}\right\rangle_{\mathrm{CV}}$ in the unbiased case. The asymptotic analysis discussed below shows that $\hat{H}_{n}(m) \simeq-m / \sqrt{6 n}$ (see $(6.22)$ and (6.25)). Then, for $j>i \gg 1$,

$$
\left\langle S_{i} S_{j}\right\rangle_{\mathrm{CV}} \simeq-\frac{1}{\sqrt{6 j}}\left(1+\sum_{k<i}\left\langle S_{k} S_{i}\right\rangle_{\mathrm{CV}}+\sum_{i<k<j}\left\langle S_{i} S_{k}\right\rangle_{\mathrm{CV}}\right) .
$$

If we again assume the scaling form $\left\langle S_{i} S_{j}\right\rangle_{\mathrm{CV}}=i^{-1 / 2} \hat{F}\left((j-i) / i^{1 / 2}\right)$ (compare (5.2)), then (6.15) leads to

$$
\hat{F}(x)=-\frac{1}{\sqrt{6}}\left(1+\int_{-\infty}^{x} \hat{F}(t) \mathrm{d} t\right)
$$

with solution

$$
\hat{F}(x)=-\frac{1}{2 \sqrt{6}} \exp (-|x| / \sqrt{6})
$$

where we have used the fact that $\hat{F}(x)$ is even.

In fact, as discussed in section 5 , both computation and simulation indicate that the correlation function in the true model is Gaussian rather than exponential. Moreover, in some related models which can be solved more completely (see appendix 3 ) we find that the recursive scheme based only on (6.6) yields correct predictions for the distribution of $M_{n}$, while the approximate measure determined by $(6.12)$ is very far from the true measure. This suggests that the restricted assumption (6.6) is more nearly correct than (6.12); it may in fact be asymptotically correct. 


\subsection{The asymptotic limit in the unbiased case}

We can extract the asymptotic behaviour of this model by passing to a continuum limit. We consider first the unbiased case $\lambda_{+}=\lambda_{-}=1$; our basic assumption is that, for $n$ large, the functions $\hat{H}, \hat{U}$ and $\hat{W}$ are slowly varying functions of $n$ and $m$ and are well approximated by smooth functions $h(t, x), u(t, x)$ and $w(t, x)$ under an appropriate scaling:

$$
\hat{H}_{n}(m) \simeq h\left(\varepsilon^{4} n, \varepsilon m\right) \quad \hat{U}_{n}(m) \approx u\left(\varepsilon^{4} n, \varepsilon m\right) \quad \hat{W}_{n}(m) \simeq w\left(\varepsilon^{4} n, \varepsilon m\right) .
$$

The relative scaling of the $x$ and $t$ variables here is forced by the requirement that the continuum function $w$ satisfies a non-trivial partial differential equation, as derived below.

We now rewrite the recurrence equations in terms of these continuum variables. Considering first the evolution equation (6.10) for $\hat{U}$, we write

$$
\begin{aligned}
& \hat{U}_{n}(m \pm 1)=u(t, x) \pm \varepsilon \partial_{x} u(t, x)+\frac{\varepsilon^{2}}{2} \partial_{x}^{2} u(t, x) \\
& \hat{U}_{n+1}(m)=u(t, x)+\varepsilon^{4} \partial_{t} u(t, x)
\end{aligned}
$$

where $t=\varepsilon^{4} n$ and $x=\varepsilon m$, and expand in powers of $\varepsilon$. Up to second order, (6.10) becomes

$$
u=\frac{1}{2}(u+w)+\varepsilon^{2}\left(\frac{\partial_{x}^{2} u}{4}-\frac{\left(\partial_{x} u\right)^{2}}{2(u+w)}\right) .
$$

To lowest order in $\varepsilon, u=w$, and, to second order,

$$
u=w+\frac{\varepsilon^{2}}{2}\left(\partial_{x}^{2} w-\frac{\left(\partial_{x} w\right)^{2}}{w}\right) .
$$

Using (6.21) and a similar analysis, (6.9) becomes, to third order,

$$
w h=\varepsilon \frac{\partial_{x} w}{w}+\varepsilon^{3}\left[\frac{\partial_{x}^{3} w}{3}-\frac{3}{8} \partial_{x}\left(\frac{\left(\partial_{x} w\right)^{2}}{2}\right)\right] .
$$

Finally, to fourth order, (6.4) becomes

$$
w+\varepsilon^{4} \partial_{f} w=\left(w+\frac{\varepsilon^{2}}{2} \partial_{x}^{2} w+\frac{\varepsilon^{4}}{24} \partial_{x}^{4} w\right)-\left(\varepsilon \partial_{x}(h w)+\frac{\varepsilon^{3}}{6} \partial_{x}^{3}(h w)\right)
$$

When (6.22) is substituted into this equation, all terms of order $\varepsilon^{2}$ cancel (this is the basic mechanism leading to the $n^{1 / 2}$ growth in $\left.\left\langle M_{n}^{2}\right\rangle_{\mathrm{CV}}\right)$, and the fourth-order terms yield

$$
\partial_{t} w=-\frac{3}{8} \partial_{x}^{2}\left[w \partial_{x}^{2}(\log w)\right]
$$

This is the fundamental evolution equation of the continuum theory in the unbiased case.

Equation (6.24) has among its solutions the Gaussian scaling solution

$$
w_{\mathrm{G}}(t, x)=(2 \pi \gamma(t))^{-1 / 2} \exp \left(-x^{2} / 2 \gamma(t)\right) \quad \gamma(t)=\sqrt{3 t / 2}
$$

which appears to be the physically relevant solution, since the initial value $w_{\mathrm{G}}(\overline{0}, x)=$ $\delta(x)$ corresponds to $\hat{W}_{0}(m)=\delta_{m 0}$. (For further discussion of solutions of this equation, see [20].) The continuum theory thus predicts that asymptotically

$$
\left\langle M_{n}^{2}\right\rangle_{\mathrm{CV}} \sim \sqrt{3 n / 2} \text {. }
$$


In table 1 we include these continuum values for comparison with values of $\left\langle M_{n}^{2}\right\rangle_{\mathrm{CV}}$ computed directly from the recursion. The agreement is excellent, and in fact the table suggests that

$$
\left\langle M_{n}^{2}\right\rangle_{\mathrm{CV}} \simeq \sqrt{3 n / 2}-\beta
$$

with $\beta \approx 1.8$.

\subsection{The asymptotic limit in the biased case}

We now turn to the asymptotic limit of the recursion scheme in the biased case. Here the mean magnetization $\left\langle M_{n}\right\rangle_{\mathrm{CV}} \equiv \Sigma_{m} m \hat{W}_{n}(m)$ will increase linearly with $n:\left\langle M_{n}\right\rangle_{\mathrm{CV}} \sim$ $\mu n$, where $\mu$ is the asymptotic single-spin magnetization (2.8); $\left\langle M_{n}^{2}\right\rangle_{\mathrm{CV}}-\left\langle M_{n}\right\rangle_{\mathrm{CV}}^{2}$ will again increase sublinearly. The correct scaling is again determined by the requirement of a non-trivial continuum partial differential equation, and continuum variables may be introduced through

$$
\begin{aligned}
& \hat{H}_{n}(m) \simeq \mu+h\left(\varepsilon^{3} n, \varepsilon(m-\mu n)\right) \\
& \hat{U}_{n}(m) \simeq u\left(\varepsilon^{3} n, \varepsilon(m-\mu n)\right) \\
& \hat{W}_{n}(m) \simeq w\left(\varepsilon^{3} n, \varepsilon(m-\mu n)\right) .
\end{aligned}
$$

An analysis of the recurrence relations in terms of these variables may be carried through along the lines above. (6.21) becomes

$$
u=\sqrt{\lambda_{+} \lambda_{-}}\left[w+\frac{\varepsilon^{2}}{2}\left(\partial_{x}^{2} w-\frac{\left(\partial_{x} w\right)^{2}}{w}\right)\right] .
$$

We now need the analogue of (6.22) only to second order:

$$
w h=2 \varepsilon C \partial_{x} w-\varepsilon^{2} \mu C\left(2 \partial_{x}^{2} w-\frac{\left(\partial_{x} w\right)^{2}}{w}\right)
$$

where $C=\sqrt{\lambda_{+} \lambda_{-}} /\left(\sqrt{\lambda_{+}}+\sqrt{\lambda_{-}}\right)^{2}$. Finally, we find from (6.4) that $w$ satisfies the thirdorder evolution equation

$$
\partial_{i} w=\mu C\left[\frac{4}{3} \partial_{x}^{3} w-\partial_{x}\left(\frac{\left(\partial_{x} w\right)^{2}}{w}\right)\right] .
$$

This is the fundamental continuum equation in the biased case.

Equation (6.31) may be linearized by the substitution $w(t, x)=v(t, x)^{2}$, to obtain

$$
3 \partial_{t} v=4 \mu C \partial_{x}^{3} v \text {. }
$$

Since (6.32) is linear we may certainly solve it, for example by the Fourier transform, and thus find $w$ at any time. Alternatively, we may note that (6.31) remains unchanged if $x$ is scaled by a factor $\gamma, t$ is scaled by $\gamma^{3}$ and $w$ is scaled by an arbitrary function of $\gamma$, and thus look for a scaling solution $w(t, x)=t^{-1 / 3} G\left(t^{-1 / 3} x\right)$; here the factor $t^{-1 / 3}$ is chosen to keep the integral $\int w(t, x) \mathrm{d} x$ normalized. $G$ must satisfy

$$
(y G(y))^{\prime}=\mu C\left(3 \frac{G^{\prime}(y)^{2}}{G(y)}-4 G^{\prime \prime}(y)\right)^{\prime} .
$$

This equation may be integrated once (with integration constant set to zero), and then the substitution $G(y)=g(y)^{4}$ yields the linear equation

$$
\rho g^{\prime \prime}(y)=-y g(y)
$$


with $\rho=16 \mu C$. The solutions of (6.34) are multiples of Airy functions $\operatorname{Ai}\left(-\rho^{-1 / 3} y\right)$, which (we take $\mu>0$ from here on, for definiteness) vanish exponentially for $y \rightarrow-\infty$ and oscillate for $y>0$. Comparison of this continuum solution with explicit calculations from the recurrence relations shows that the physically relevant solution of $(6.34)$ is

$$
g(y)= \begin{cases}c \mathrm{Ai}\left(-\rho^{-1 / 3} y\right) & \text { if } y<y_{0} \\ 0 & \text { if } y \geqslant y_{0} .\end{cases}
$$

where $y_{0}=-\rho^{1 / 3} z_{0}$, with $z_{0}<0$ the largest zero of the Airy function, and $c$ is a normalization constant. In fact, it appears that, on the scale determined by $(6.28)$, $h(x) \rightarrow \infty$ as $x \rightarrow t^{1 / 3} y_{0}$, and hence asymptotically $\hat{W}_{n}(m) \equiv 0$ for $m>\mu n+n^{1 / 3} y_{0}$. The solution of the recurrence relations approaches the asymptotic regime very slowly, so that even for the largest systems we have studied the continuum theory does not accurately predict $\left\langle M_{n}\right\rangle_{\mathrm{CV}}$ and $\left\langle M_{n}^{2}\right\rangle_{\mathrm{CV}}$; however, if the distribution $\hat{W}_{n}(m)$ is normalized in variance and mean, it agrees very well with $g\left(n^{-1 / 3} m\right)^{4}$, similarly normalized.

\section{Continuum field theory}

The large-scale properties of interfaces can often be captured through a time-dependent continuum field theory of the Ginzburg-Landau type [14]. When applicable, these theories provide both simple physical insights and powerful computational tools for leading asymptotic behaviour. For growth processes and interfaces driven by chemical potential differences this approach was implemented by Kardar, Parisi and Zhang (KPZ) [15] (see also the review [16]). In this section we develop the method for the Toom interface as it will turn out to be a novel and somewhat unusual example from the general point of view. We also compare the predictions of the continuum theory with the more microscopic approach developed before.

Our starting point is the velocity of an infinite, macroscopically flat interface between the + and - phase. As an example, we plot in figure 8 the normal velocity $u(\theta)$ at zero noise and at two levels of unbiased noise, $p=q=0.04$ and $p=q=0.065$; our convention is that the normal vector to the interface is oriented from the + to the - phase and that $\theta$ is the angle between this normal and due north (the positive $j$-axis). At zero noise the interface velocity is obtained by explicit computation from the Toom dynamics. At finite noise no computation seems feasible; finite noise velocities in figure 8 have been obtained from simulations (For $0<\theta<\pi / 2$ an approximate interface velocity at low noise was computed in section 2 , $\mathrm{cf}(2.7)$; it should be noted that this low-noise approximation is not uniform in $\theta$ and is optimal for $\theta \simeq \pi / 4$.)

Of course, symmetries of the Toom model are reflected in symmetry properties of the velocity function $u(\theta)$. The model has an alternate formulation on a spacetime lattice whose constant-time cross-sections are triangular lattices [8]; in this formulation, it is clear that the model has the symmetry group $\mathrm{D}_{3}$ of an equilateral triangle. In our NEC formulation this symmetry group is generated by two transformations of the square lattice: the reflection across the diagonal, $(i, j) \rightarrow(j, i)$, and the time-dependent linear transformation $(i, j) \rightarrow(j,-(i+j+t))$, which corresponds in the alternate formulation to a $2 \pi / 3$ rotation of the triangular lattice. That is, if $\sigma_{i j}(t)$ satisfies the Toom dynamics (1.1), then so do $\sigma_{j i}(t)$ and $\sigma_{j,-(i+j+t)}(t)$, as may be checked by explicit calculation. Because the symmetry group involves time-dependent transformations, it relates interfaces which move at different velocities. The 'rotation' symmetry given above, for example, carries an interface with $\theta=\pi / 4$ to one with $\theta=\theta_{1}$, where $\theta_{1} \in[\pi / 2, \pi]$ 


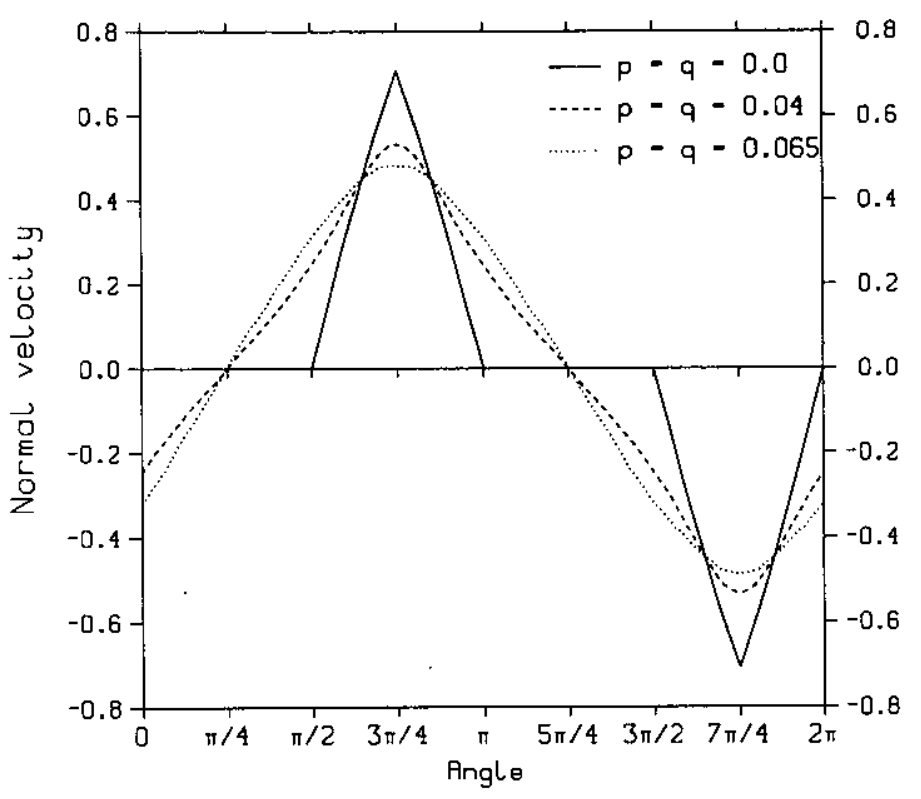

Figure 8. Angular dependence of normal interface velocity in unbiased case.

satisfies $\tan \theta_{1}=-\frac{1}{2}$. If the noise is unbiased, then $u(\pi / 4)=0$, and from the full symmetry group $\mathrm{D}_{3}$ we find that $u(5 \pi / 4)=0$ and

$$
u\left(\theta_{1}\right)=u\left(3 \pi / 2-\theta_{1}\right)=-u\left(\theta_{1}+\pi\right)=-u\left(5 \pi / 2-\theta_{1}\right)=1 / \sqrt{5} \text {. }
$$

We will study both the time development of an infinite, initially flat interface, for which the initial interface position defines a reference line, and the anchored interface, for which we choose the average interface position as reference line. In each case we introduce a coordinate $x$ along this line, oriented so that travelling in the direction of increasing $x$ puts the + phase to the right; note that by our conventions the anchored interface corresponds to angle $\theta \in[\pi, 3 \pi / 2]$, and the reference line is oriented away from the origin. Figure 1 suggests that overhangs may be ignored; the interface height at time $t$, when averaged over a few lattice spacings, is then a single-valued function $x \rightarrow h_{t}(x)$. It is reasonable to assume that, viewed on a large scale, every surface element moves with the normal velocity of an infinite interface having the same slope. If $\hat{u}\left(h^{\prime}\right)$ denotes the interface velocity normal to the reference line and written as a function of the slope $\partial h / \partial x \equiv h^{\prime}$, the interface then is governed by the equation

$$
\partial_{t} h_{t}(x)=\hat{u}\left(\partial_{x} h_{r}(x)\right) .
$$

To include fluctuation effects the KPZ approach is to add noise and dissipation to (7.2) and to replace $\hat{u}\left(h^{\prime}\right)$ by a polynomial of degree determined by the relevant order. This yields

$$
\partial_{t} h_{t}=v_{0}+v_{1} \partial_{x} h_{t}+\frac{1}{2} v_{2}\left(\partial_{x} h_{t}\right)^{2}+\frac{1}{6} v_{3}\left(\partial_{x} h\right)^{3}+D \partial_{x}^{2} h_{t}+\sqrt{\sigma} \zeta_{t}
$$

Here $\zeta_{1}$ is normalized Gaussian white noise,

$$
\left\langle\zeta_{t}(x) \zeta_{1} \cdot\left(x^{\prime}\right)\right\rangle=\delta\left(t-t^{\prime}\right) \delta\left(x-x^{\prime}\right) .
$$

Simple power counting shows that higher-order nonlinearities are irrelevant on a large scale. The cubic nonlinearity scales as the noise and the dissipation and is thus marginal. 
It is tempting to choose $v_{j}, j=0, \ldots, 3$, as the Taylor coefficients of $\hat{u}\left(h^{\prime}\right)$ at $h^{\prime}=0$, but in fact the Laplacian and noise terms in (7.3) will modify the interface velocity and thus renormalize these bare values. This will give rise to effective parameters in (7.3) which we can identify only through comparing its large-scale properties with those of the Toom dynamics.

Equations (7.3) and (7.4) must be supplemented by initial and boundary conditions. To study the time development of the initially flat interface we take $h_{0}(x)=0$ on the entire line or on an interval with periodic boundary conditions. In the anchored case, the interface must pass through the origin; hence $x \geqslant 0$ and

$$
h_{t}(0)=0 \text {. }
$$

For the moment we discuss the non-stationary problem; we will return to the anchored interface shortly.

If $v_{2} \neq 0$ in (7.3), then the quadratic term $\left(h^{\prime}\right)^{2}$ dominates; $(7.3)$ is then equivalent to the noise-driven Burgers equation studied in [17]. In particular, the average width of the interface grows as

$$
\left\langle h_{t}(x)^{2}\right\rangle^{1 / 2} \simeq\left(v_{2} \chi^{2} t\right)^{1 / 3}
$$

for large $t$. Here

$$
\chi=\frac{\sigma}{2 D}
$$

is the strength of the stationary covariance for $h^{\prime},\left\langle h^{\prime}(x) h^{\prime}(y)\right\rangle=\chi \delta(x-y)$. The prefactor in (7.6) is not known with accuracy (it is given by an integral over the scaling function; see [16], section 6.1 ). The $t^{1 / 3}$ growth has been confirmed for a large variety of systems [16] and there is little doubt that it is the correct asymptotics.

Now, a least if we identify $v_{2}$ with its bare value $\hat{u}^{\prime \prime}(0)$, figure 8 suggests that for almost all inclinations we have $v_{2} \neq 0$ and hence a $t^{1 / 3}$ widening of the interface. The prominent exception is of course the interface along the diagonal (for symmetric noise $p=q$ ); there we must have $v_{0}=v_{2}=0$ because of the +- symmetry, and figure 8 and the low-noise approximation (2.7) suggest that $v_{1}$ and $v_{3}$ do not vanish. A complete discussion of the interface at this inclination would include the effect of the $h^{\prime 3}$ nonlinearity. We have not carried out such an analysis, and the nature of the effect is not clear: a mode coupling theory in the spirit of [18] indicates logarithmic corrections to the linear theory, but it is of course possible that the cubic term is marginally relevant, i.e. that the fixed point at zero coupling is unstable under this perturbation. In any case, for comparison with our previous approximations it is already instructive to investigate the linear theory in which $v_{3}=0$. Then (7.3) becomes

$$
\partial_{t} h_{t}=-v \partial_{x} h_{t}+D \partial_{x}^{2} h_{t}+\sqrt{\sigma} \zeta_{t}
$$

where we have written $v \equiv-v_{1}$ for notational convenience. For initial condition $h_{0}(x)=$ 0 the interface broadens as

$$
\begin{aligned}
\left\langle h_{t}(x)^{2}\right\rangle^{1 / 2} & =\left(\sigma \int_{0}^{t} \frac{\mathrm{d} s}{2 \pi} \int \mathrm{d} k \mathrm{e}^{-2 D k^{2} s}\right)^{1 / 2} \\
& =\chi^{1 / 2}(2 D t / \pi)^{1 / 4} .
\end{aligned}
$$

We now turn to the anchored interface, and argue that the time-dependent broadening according to (7.6) and (7.9) reappears as static broadening in the anchored case. 
We first note that stationarity requires the interface velocity to vanish; with our convention on the reference line this implies that $v_{0}=0$. In particular, in the unbiased case $p=q$ the average interface has to be along the diagonal: $\theta=5 \pi / 4$. Secondly, we have $v \equiv-v_{1}>0$; cf figure 8 . This means that excitations travel outwards, of course satisfying the boundary condition $h_{t}(0)=0$. Thus the stationary problem is comparable to the non-stationary one provided that we identify space and time through $x=v t$. On the basis of this analogy we arrive at the following predictions for the interface fluctuations.

In the unbiased case,

$$
\left\langle h(x)^{2}\right\rangle^{1 / 2}=\chi^{1 / 2}(2 / \pi)^{1 / 4}(\xi x)^{1 / 4}
$$

for large $x$; here we have introduced the length

$$
\xi=\frac{D}{v}
$$

The exponent in (7.10) is close to the exponent $\nu=0.265$ observed in simulations of the spin dynamics, and is the same as that obtained from the collective variable approximation (see (6.26)), but the identification of the parameters in (7.10) with those of the Toom model, carried out below, shows that the prefactor is different. (7.10) agrees, even up to prefactor, with the result derived in appendix 1 from stationarity conditions plus some additional assumptions; we will see shortly that this agreement is not so surprising. If the cubic nonlinearity modifies the large time behaviour in (7.9), this will be reflected in a corresponding modification of (7.10).

In the biased case (7.6) translates to

$$
\left\langle h(x)^{2}\right)^{1 / 2} \simeq\left(v_{2} \chi^{2} x / v\right)^{1 / 3}
$$

where we do not know the prefactor with accuracy. Thus, for the biased dynamics we obtain the $x^{1 / 3}$ growth. Very remarkably, this scaling is reproduced by the collective variable approximation, which seems quite different from the KPZ approach.

The nonlinearities in (7.3) make it difficult to go beyond a heuristic relation between time-dependent broadening and anchored interface fluctuations. However, for the linear theory, which applies only to the unbiased interface, we can check the picture in more detail and also compute stationary correlations. The problem is to determine the stationary measure of (7.8), with $x \geqslant 0, v>0$, and with boundary condition $h_{r}(0)=0$. By linearity the stationary measure is Gaussian with mean zero. To compute the covariance, let us define

$$
p_{f}\left(x, x^{\prime}\right)=(4 \pi D t)^{-1 / 2}\left(\mathrm{e}^{-\left\langle x^{i}-x-v t i^{2} / 4 \bar{D} t\right.}-\mathrm{e}^{\left.-i x^{i}+x-v t\right)^{2} / 4 \bar{D} t} \mathrm{e}^{-v x / \bar{D}}\right)
$$

to be the transition probability from $x$ to $x^{\prime}$ in time $t$ for the diffusion process generated by $v \partial_{x}+D \partial_{x}^{2}$ with zero boundary condition at $x=0$, that is, $p_{t}$ is the transition probability for a diffusing particle with constant drift $v$ to the right and with absorbing boundary condition at zero. The stationary height-height correlation is then given by

$$
\begin{aligned}
\langle h(x) h(x+y)\rangle & =\sigma \int_{0}^{\infty} \mathrm{d} t \int_{0}^{\infty} \mathrm{d} x^{\prime} p_{t}\left(x^{\prime}, x\right) p_{t}\left(x^{\prime}, x+y\right) \\
& =\frac{\sigma}{2 \pi} \int_{-\infty}^{\infty} \mathrm{d} \lambda \int_{0}^{\infty} \mathrm{d} x^{\prime} R_{i \lambda}\left(x^{\prime}, x\right) R_{-i \lambda}\left(x^{\prime}, x+y\right)
\end{aligned}
$$


where

$$
R_{z}\left(x^{\prime}, x\right)=\int_{0}^{\infty} \mathrm{d} t \mathrm{e}^{-z t} p_{t}\left(x^{\prime}, x\right)
$$

is the resolvent. Explicitly,

$$
R_{z}\left(x, x^{\prime}\right)= \begin{cases}\left(v^{2}+4 z v\right)^{-1 / 2} \mathrm{e}^{-\alpha_{+} x}\left(\mathrm{e}^{\alpha_{+} x^{\prime}}-\mathrm{e}^{\alpha_{-} x^{\prime}}\right) & \text { for } 0 \leqslant x^{\prime}<x \\ \left(v^{2}+4 z v\right)^{-1 / 2} \mathrm{e}^{-\alpha_{-} x^{\prime}}\left(\mathrm{e}^{-\alpha_{-} x}-\mathrm{e}^{-\alpha_{+} x}\right) & \text { for } 0 \leqslant x \leqslant x^{\prime}\end{cases}
$$

with $\alpha_{ \pm}=(v / 2 D)\left(1 \pm\left(1+4 z D / v^{2}\right)^{1 / 2}\right), \operatorname{Re}(z)>0$. A straightforward computation yields $\langle h(x) h(x+y)\rangle$

$$
\begin{aligned}
= & \frac{\chi \xi}{\pi} \int \mathrm{d} \lambda\left(\frac{1}{2 \lambda^{2}}\left(1-\mathrm{e}^{(1-a) x / \xi}\right) \mathrm{e}^{(1-a) y / 2 \xi} \cos (y \lambda / \xi a)+\left(1+16 \lambda^{2}\right)^{-1 / 2}\right. \\
& \times 2 \cosh (y / 2 \xi)\{r[(2 x+y) / \xi]-r(y / \xi)]\})
\end{aligned}
$$

with

$r(y)=\mathrm{e}^{-a y / 2}\left\{\left[\left(1+a-a^{2}\right) / a^{2}(1+a)\right] \cos (y \lambda / a)-\left(2 \lambda / a^{3}\right) \sin (y \lambda / a)\right\}$

and

$$
a=\left\{\left[1+\left(1+16 \lambda^{2}\right)^{1 / 2}\right] / 2\right\}^{1 / 2} .
$$

For large $x$ the integral is dominated by the first term at small $\lambda$. This yields

$$
\begin{aligned}
\left\langle h(x)^{2}\right\rangle & =\frac{\chi}{\pi} \sqrt{\xi x} \int \mathrm{d} \lambda\left(1 / 2 \lambda^{2}\right)\left[1-\exp \left(-2 \lambda^{2}\right)\right] \\
& =\chi \sqrt{2 \xi x / \pi} .
\end{aligned}
$$

Note that this result agrees with (7.10), giving support to the heuristic method used there. For the height difference correlation one obtains

$$
\left\langle[h(x)-h(x+y)]^{2}\right\rangle=\chi \sqrt{x \xi} g(y / \sqrt{x \xi})
$$

for $x$ large and $y$ of the order of $\sqrt{x}$, where the scaling function $g$ is given by

$$
g(w)=\frac{1}{\pi} \int \mathrm{d} \lambda \lambda^{-2}(1-\cos w \lambda)\left[1-\exp \left(-2 \lambda^{2}\right)\right] .
$$

It is of interest to compute also the slope-slope correlations. We differentiate (7.17) and take the scaling limit. Then

$$
\left\langle h^{\prime}(x) h^{\prime}(x+y)\right\rangle=\chi\left[\xi \delta(\xi y)-(8 \pi \xi x)^{-1 / 2} \exp \left(-y^{2} / 8 \xi x\right)\right]
$$

for $x$ large and $y$ of the order of $\sqrt{x}$. In fact, (7.23) is just the second derivative of the scaling function in (7.21).

The slope-slope correlation (7.23) agrees with the spin-spin correlation found in section 5 (see (5.8)). Actually this is not so surprising, once we consider the stationarity condition in differential form. It reads

$$
\left(-v \partial_{x}+D \partial_{x}^{2}-v \partial_{y}+D \partial_{y}^{2}\right)\left\langle h^{\prime}(x) h^{\prime}(y)\right\rangle=\sigma \partial_{x} \partial_{y} \delta(x-y) .
$$


To compare this equation with (5.1) we note that the latter resembles (7.24) in the limit in which $\left\langle S_{i} S_{j}\right\rangle$ is a slowly varying function of $i$ and $j$. Expanding to second order in (5.1) yields the homogeneous form of (7.24), with $\xi=D / v=\frac{3}{2}$. Furthermore, $\chi=1$ since the steady state on the ring is uncorrelated. With these identifications, (7.23) coincides with (5.8), and (7.10) with (A1.9) and (A1.10).

In summary, the continuum theory of the Toom interface teaches us two novel aspects: (i) the steady-state fluctuations of an interface anchored at the origin and the non-stationary broadening of an initially flat interface are governed by the same scaling exponent provided that fluctuations travel outward $(v>0)$; (ii) for unbiased noise, the symmetry of the Toom dynamics implies that the interface along the diagonal is described by a continuum theory with a $h^{\prime 3}$ nonlinearity only.

We ask finally whether the properties found here for the Toom interfaces are special to the NEC majority rule or are of a more generic nature. We have in mind here $2 \mathrm{D}$ spin-flip dynamics with no conservation law and with a local updating rule, as in the Toom model. Furthermore, the model should be ferromagnetic in spirit and should be at a low noise level (or low temperature), which roughly means that with high probability a spin follows its neighbours. An example is the stochastic Ising model at phase coexistence, i.e. with zero external magnetic field.

It is clear from the analysis given above that, as far as the continuum theory is concerned, the only relevant property is the form of the macroscopic inclinationdependent interface velocity. If its second derivative does not vanish, then the standard KPZ scaling theory applies (dynamic exponent $z=\frac{3}{2}$, static exponent $\zeta=\frac{1}{2}$ in the two dimensions). If for a particular orientation of the interface the second derivative vanishes but the third does not, then the model is in the universality class of the diagonal interface in the Toom model with symmetric noise. If both the second and third derivatives vanish, then the universality class is that of an equilibrium interface (dynamic exponent $z=2$ ). The sign (or vanishing) of the first derivative will affect stationary fuctuations in an anchored interface, as in the Toom case. Thus, we expect that for a probabilistic cellular automaton with (i) two stable phases and (ii) nonvanishing interface velocity, the large-scale fluctuations of an interface will be identical to those obtained here for the Toom model.

\section{Conclusion}

In this paper we have discussed two general approaches to the behaviour of the anchored interface in the $2 \mathrm{D}$ Toom model. The first is a reformulation of the problem, in the limit of very low noise, as the dynamics of a 1D spin model; the width of the interface in the $2 \mathrm{D}$ Toom model is proportional to the magnetization of the $1 \mathrm{D}$ spin model. We have studied the $1 \mathrm{D}$ model through the stationarity relations valid in the steady state, and through the collective variable approximation.

An analytic expression for the scaling form of the pair correlation was derived from the stationarity relations, supplemented with the assumptions that such a scaling form exists and that in the asymptotic regime the four-point functions are much smaller than pair correlations. The resulting expression (5.8) is in good agreement with the numerical data; since we could not find a clean way of justifying the assumptions needed to derive it, however, we cannot be confident of its validity. Moreover, we are not yet able to calculate other quantities in the asymptotic regime, primarily because such calculations require additional assumptions, which we could not formulate in a 
way consistent with the stationarity conditions that we know. The problem of the asymptotic behaviour of the 1D spin model is thus still open.

The collective variable approximation to the $1 \mathrm{D}$ model yields the prediction that the width of the interface is of order $L^{1 / 4}$ in the unbiased case and $L^{1 / 3}$ in the biased case. Our numerical results are not inconsistent with these predictions. In the unbiased case, in fact, the agreement is at the moment tantalizingly good, even to the prefactor, but we cannot be sure that our simulations have reached their asymptotic regime. The simulations in the biased case appear to be sufficiently far from asymptopia that even tentative conclusions are not possible.

Our second approach to the interface problem is to relate it to the well-studied theory of time dependence of fluctuations in growing interfaces. The key idea is that the translational velocity of excitations along the interface converts a $t^{\alpha}$ growth of fluctuations in time to a $x^{\alpha}$ growth of fluctuations along the interface. (With an appropriate choice of boundary conditions this phenomenon can be also observed in other interface problems; see [21] for a discussion of the case of Eden growth.) It is striking that (up to the question of proper treatment of a marginal nonlinearity) this theory predicts the same exponents of $\frac{1}{4}$ and $\frac{1}{3}$ as does the collective variable approximation to the iD theory.

We finally remark that the iD model, a spin model which evolves according to a very simple dynamical rule, is rather similar to a large class of problems which have been studied recently under the name of self-organized criticality. It would be interesting to know if there is a closer connection with these problems, and if some of the methods used in the present work (in particular our collective variable approach) could be of some use to understand these other problems defined only by dynamical rules.

\section{Appendix 1. Growth of $\left\langle M_{n}^{2}\right\rangle$}

In this appendix, we shall obtain an expression for $\left\langle M_{n}^{2}\right\rangle$ by starting from some stationarity conditions and making assumptions (A1.3)-(A1.4) and (A1.9) for the correlation functions.

From the stationarity condition (4.7), one can derive the following identity:

$$
2\left\langle M_{n-1} S_{n}\right\rangle=-\frac{\left\langle K_{n-1}\right\rangle}{2}-\frac{\left\langle K_{n-1} S_{n} M_{n-1}\right\rangle}{2}+\left\langle M_{n-1} S_{n}\right\rangle
$$

By replacing $K_{n-1}$ by its expansion (4.4) and neglecting all correlation functions of order higher than four we obtain

$$
\begin{aligned}
2\left\langle M_{n-1} S_{n}\right\rangle=- & -\sum_{p>q \geqslant 0} \frac{\left\langle S_{n-1-p} S_{n-1-q}\right\rangle+\left\langle S_{n} S_{n-1-q}\right\rangle+\left\langle S_{n} S_{n-1-p}\right\rangle}{2^{p}} \\
& -\sum_{p>q \geqslant 0} \sum_{r \neq p ; r \neq q} \frac{\left\langle S_{n-1-p} S_{n-1-q} S_{n-1-r} S_{n}\right\rangle}{2^{p}} \\
& -\sum_{p>q>r>s \geqslant 0} \frac{1}{2^{p}}\left(\left\langle S_{n-1-p} S_{n-1-q} S_{n-1-r} S_{n-1-s}\right\rangle\right. \\
& +\left\langle S_{n-1-p} S_{n-1-q} S_{n-1-r} S_{n}\right\rangle+\left\langle S_{n-1-p} S_{n-1-q} S_{n} S_{n-1-s}\right\rangle \\
& \left.+\left\langle S_{n-1-p} S_{n} S_{n-1-r} S_{n-1-s}\right\rangle+\left\langle S_{n} S_{n-1-q} S_{n-1-r} S_{n-1-s}\right\rangle\right)
\end{aligned}
$$


Then assuming that for large $i, j, k, l$, the pair correlation function satisfies the scaling form (5.2),

$$
\left\langle S_{i} S_{j}\right\rangle \approx \frac{1}{i^{1 / 2}} F\left(\frac{j-i}{i^{1 / 2}}\right)
$$

and that the four-point function satisfies

$$
\left\langle S_{i} S_{j} S_{k} S_{l}\right\rangle \simeq \frac{1}{i} G\left(\frac{j-i}{i^{1 / 2}}, \frac{k-i}{i^{1 / 2}}, \frac{l-i}{i^{1 / 2}}\right)
$$

one gets from (A1.2) to leading order

$$
2\left\langle M_{n-1} S_{n}\right\rangle+1=n^{-1 / 2}\left(-6 F(0)-2 \int_{0}^{\infty} G(-x, 0,0) \mathrm{d} x\right) .
$$

Then using the fact that

$$
\left\langle M_{n}^{2}\right\rangle-\left\langle M_{n-1}^{2}\right\rangle=2\left\langle M_{n-1} S_{n}\right\rangle+1
$$

one gets for the growth of $\left\langle M_{n}^{2}\right\rangle$

$$
\left\langle M_{n}^{2}\right\rangle \simeq \alpha n^{1 / 2}
$$

with

$$
\alpha=-12 F(0)-4 \int_{0}^{\infty} G(-x, 0,0) \mathrm{d} x .
$$

So we see that the calculation of the leading order of $\left\langle M_{n}^{2}\right\rangle$ requires knowledge of the four-point function (or alternatively the correction to the scaling form (5.2)).

To determine this four-point function, we only know the stationarity relation (4.13); the problem is to choose the solution of these equations. If we assume that in the asymptotic regime the factorization

$$
\left\langle S_{i} S_{j} S_{k} S_{l}\right\rangle \simeq\left\langle S_{i} S_{j}\right\rangle\left\langle S_{k} S_{l}\right\rangle+\left\langle S_{i} S_{k}\right\rangle\left\langle S_{j} S_{l}\right\rangle+\left\langle S_{i} S_{i}\right\rangle\left\langle S_{j} S_{k}\right\rangle
$$

is valid, then we can check that this form satisfies (4.13) and obtain for the coefficient $\alpha$ of $(\mathrm{A1.7)}$

$$
\alpha=-12 F(0)-12 F(0) \int_{0}^{\infty} F(-x) \mathrm{d} x=\sqrt{\frac{3}{\pi}}
$$

where $F$ is given by (5.8).

This result, based both on the scaling (A1.3) and (A1.4) and the factorization (A1.9), seems to give an estimate for the prefactor $\alpha$ of $\left\langle M_{n}^{2}\right\rangle$ which is too small to explain the results of our numerical simulations of section 3 . It is therefore very likely that one of these two assumptions is not valid.

\section{Appendix 2. Rigorous control of the zero-noise limit}

In this appendix we remark that the agreement of the ID spin model and the zero-noise limit of the Toom model may be established rigorously; an appropriate rescaling of time is necessary. One way to formulate the result is as follows. Fix positive rates $\lambda_{+}$, $\lambda_{-}$for the ID spin model, let $S(t)$ denote the configuration of this model at time $t$, 
and let $\bar{\sigma}(t)$ denote the configuration of a spin system on the third quadrant of the integer lattice in which the boundary between + and - spins consists precisely of the staircase associated with $S(t)$, with no + spins below or - spins above this staircase. Now, for positive and small $\varepsilon$, let $\sigma^{(\varepsilon)}(t)$ denote the configuration at (integer) time $t$ of the 2D Toom model with noise parameters $p=\varepsilon \lambda_{+}, q=\varepsilon \lambda_{-}$, and let $\bar{\sigma}^{(\varepsilon)}(t)$ be the same process with a rescaled, continuous time:

$$
\bar{\sigma}^{(\varepsilon)}(t)=\sigma^{(\varepsilon)}(\llbracket t / \varepsilon \rrbracket)
$$

where $\llbracket s \rrbracket$ is the greatest integer not exceeding $s$. Then $S(t)$ and the processes $\sigma^{(\varepsilon)}(t)$, for all values of $\varepsilon$, may be defined on the same probability space $\Omega$, in such a way that for almost all $(t, \omega) \in \mathbb{R}_{+} \times \Omega$ and any finite region $\Lambda$,

$$
\left.\lim _{\varepsilon \rightarrow 0} \bar{\sigma}^{(\varepsilon)}(t)(\omega)\right|_{\Lambda}=\left.\bar{\sigma}(t)(\omega)\right|_{\Lambda}
$$

\section{Appendix 3. Related spin exchange models}

In this appendix we consider a family of $1 \mathrm{D}$ spin exchange models which are simple modifications of the model introduced in section 2 . The new models are in some cases more tractable than the original and serve to illuminate the relation between the original model and the collective variable approximation of section 6 .

Let $\mathcal{M}$ denote the $1 D$ spin exchange model introduced in section 2 ; we will consider here only the unbiased case $\lambda=1$, although the generalization to arbitrary $\lambda$ is straightforward. We define a model $\mathcal{M}^{(k)}$ by modifying the dynamics of $\mathcal{M}$ : in $\mathcal{M}^{(k)}$, only the $k$ left-most spins in any block of + or - spins in a configuration may exchange or flip; as in $\mathcal{M}$, an exchange is with the first spin to the right having opposite sign and a flip occurs in a finite system which no such spin exists. Clearly, $\mathscr{M}$ corresponds to $k=\infty$. The stationarity relation (4.8) becomes (writing \langle\rangle$_{k}$ for expectations in $\mathcal{M}^{(k)}$ )

$$
\left\langle K_{n}^{(k)} S_{n} \phi\left(M_{n-1}\right)\right\rangle_{k}=0
$$

where $\phi$ is any function and $K_{n}^{(k)}=\min \left\{k, K_{n}\right\}$.

We first consider the case $k=1$, for which $K^{(k)} \equiv 1$. Then (A3.1) implies that $S_{n}$ is independent of $\dot{M}_{n-1}$; it is an immediate consequence that the distribution of $\dot{M}_{n}$ is given by

$$
W_{n}^{(1)}(m)=\left(\begin{array}{c}
n \\
(n+m) / 2
\end{array}\right)
$$

i.e. that it is the same as if the measure on spin configurations were Bernoulli with magnetization zero. Thus

$$
\left\langle M_{n}^{2}\right\rangle_{1}=n \text {. }
$$

On the other hand, the measure on spin configurations in $\mathcal{M}^{(1)}$ is in fact far from Bernoulli. The easiest way to see this is to note that the dynamics of $\mathcal{M}^{(1)}$, when implemented in the ring geometry, will eventually reduce any initial configuration in which nôt ăli șinins have the same sigñ to a configuration consisting of only two blocks. In the semi-infinite geometry new blocks are always produced at the extreme left, since the left-most spin can always exchange, but as we move to the right the consolidation effect increases the length of blocks. Simulations indicate that at a distance of $n$ links from the origin the typical block has size of order $\sqrt{n}$. 
For general $k>1$ we can say much less about the model $\mathcal{M}^{(k)}$, but it is possible to compute $\left\langle M_{n}^{2}\right\rangle_{2}$ exactly. To do so, we take $\phi\left(M_{n}\right)=M_{n}$ in (A3.1) and write $K_{n}^{(2)}=$ $\left(3+S_{n} S_{n-1}\right) / 2$ to obtain the recursion relation $3\left\langle M_{n-1} S_{n}\right\rangle_{2}+\left\langle M_{n-2} S_{n-1}\right\rangle_{2}+1=0$. The recursion is easily solved explicitly; the initial condition $\left\langle M_{1} S_{2}\right\rangle_{2}=-\frac{1}{3}$ is obtained from (2.3), which is valid here since $\mathcal{M}$ and $\mathcal{M}^{(2)}$ agree for systems of size $L \leqslant 2$. From $\left\langle M_{n}^{2}\right\rangle_{2}=\left\langle M_{n-1}^{2}\right\rangle_{2}+2\left\langle M_{n-1} S_{n}\right\rangle_{2}+1$ we then find

$$
\left\langle M_{n}^{2}\right\rangle_{2}=\frac{n}{2}+\frac{3}{8}+\frac{1}{8}\left(-\frac{1}{3}\right)^{n-1} \text {. }
$$

It is straightforward to derive a collective variable approximation for the model $\mathcal{M}^{(k)}$. We let $W_{n}^{(k)}(m)$ denote the probability in $\mathcal{M}^{(k)}$ that $M_{n}=m$, and set $H_{n}^{(k)}(m)=\left\langle S_{n+1} \mid M_{n}=m\right\rangle_{k}$ and

$$
U_{n}^{(k, j, \pm)}(m)=\left\langle K_{n}^{(j, \pm)} \mid M_{n}=m \pm 1\right\rangle_{k} W_{n}(m \pm 1)
$$

where $K_{n}^{(j \pm \pm)}=\min \left\{j, K_{n}^{ \pm}\right\}$. The argument of section 6 then leads to the introduction of approximating variables $\hat{U}^{(k, j, \pm)}, \hat{W}^{(k)}$ and $\hat{H}^{(k)}$ satisfying

$$
\begin{aligned}
& \hat{W}_{n+1}^{(k)}(m)=\frac{1}{2}\left(1+\hat{H}_{n}^{(k)}(m-1)\right) \hat{W}_{n}^{(k)}(m-1)+\frac{1}{2}\left(1-\hat{H}_{n}^{(k)}(m+1)\right) \hat{W}_{n}^{(k)}(m+1) \\
& \hat{U}_{n}^{(k, k,+)}(m)=\hat{U}_{n}^{(k, k,-)}(m)
\end{aligned}
$$

and

$$
\hat{U}_{n+i}^{(k, j, \pm)}(m)=\frac{1}{2}\left(1 \pm \hat{H}_{n}^{(k)}(m)\right)\left(\hat{W}_{n}^{(k)}(m)+\sum_{i=1}^{j-1} \hat{U}_{n}^{(k, i, \pm)}(m \mp 1)\right)
$$

The solution procedure is as before.

For $k=1$ this approach leads to $\hat{H}^{(k)} \equiv 0$ and to the correct distribution of $M_{n}$ :

$$
\hat{W}_{n}^{(1)}(m)=W_{n}^{(1)}(m)=\left(\begin{array}{c}
n \\
(n+m) / 2
\end{array}\right) .
$$

On the other hand, the collective variable measure on spin configurations given by (6.12) is just the Bernoulli measure which, as we have noted, is far from correct. It is this circumstance which suggests the possibility that in the true model $\mathcal{M}$ the collective variable approximation to the fluctuations in $M_{n}$ may be asymptotically correct, or nearly so, even though the collective variable measure clearly has the wrong two-point correlation function.

For all $k$ we may, as in section 6 , obtain an asymptotic continuum equation describing the distribution $W^{(k)}$. Here the correct scaling is $\hat{W}_{n}^{(k)}(m) \simeq w^{(k)}\left(\varepsilon^{2} n, \varepsilon m\right)$, with $w^{(k)}$ satisfying the diffusion equation: $\partial_{t} w^{(k)}=2^{-k} \partial_{t} w^{(k)}$. For $k=1$ and $k=2$ this leads to the correct asymptotic behaviour of $\left\langle M_{n}^{2}\right\rangle_{k}$ (see (A3.2), (A3.3)). The vanishing of the diffusion constant in the limit $k \rightarrow \infty$ is of course a signal that a different scaling is appropriate for the model $\mathcal{M}$.

We finally remark that the models $\mathscr{M}^{(k)}$ may be viewed as low-noise descriptions of the anchored interface in certain cellular automata to which Toom's non-ergodicity result applies. For example, for $k=1$, we may modify (1.1) as

$$
\sigma_{i, j}(t+1)= \begin{cases}\operatorname{sign}\left(\sigma_{i, j+1}(t)+\sigma_{i+1, j}(t)+\sigma_{i, j}(t)\right) & \text { with probability } 1-2 p \\ \sigma_{i+1, j+1}(t) & \text { with probability } 2 p(1-p) \\ +1 & \text { with probability } p^{2} \\ -1 & \text { with probability } p^{2}\end{cases}
$$

and then let $p \rightarrow 0$. 


\section{References}

[1] Bak P, Tang T and Wiesenfeld K 1988 Self-organized criticality Phys. Rev. A 38 364- 74

Grinstein G, Lee D H and Sachdev S 1990 Conservation laws, anisotropy, and 'self-organized criticality' in noisy nonequilibrium systems Phys. Rev. Lett. 64 1927-30

Grinstein G and Lee D H 1991 Phys. Rev. Lett. $66177-80$

[2] Garrido P L, Lebowitz J L, Maes C and Spohn H 1990 Long-range correlations for conservative dynamics Phys. Rev. A 42 1954-68

Cheng Z, Garrido P L, Lebowitz J L and Valles L 1991 Long-range correlations in stationary nonequilibrium systems with conservative anisotropic dynamics Europhys. Lett. 14507

[3] Carison J, Chayes J, Grannan E R and Swindle G H 1990 Self-organized criticality and singular diffusion Phys. Rev. Lett. 65 2547-50

[4] Stavskaya O N 1973 Math. USSR Sbornik 21395

Toom A L, Vasilyev N B, Stavskaya O N, Mitjushin L G, Kurdomov G L, and Pirogov S A 1989 Discrete local markov systems Preprint

[5] Rujàn P 1987 Cellular automata and statistical mechanical models J. Stat. Phys. 49 139-222

Georges A and LeDoussal P 1989 From equilibrium spin models to probabilistic cellular automata J. Stat. Phys. 54 1011-64

[6] Toom A L 1980 Stable and attractive trajectories in multicomponent systems Multicomponent Random Systems ed R L Dobrushin and Ya G Sinai (New York: Marcel Dekker)

[7] Bennett $C$ and Grinstein G 1985 Role of irreversibility in stabilizing complex and nonergodic behavior in local interacting discrete systems Phys. Rev. Lett. 55 657-60

[8] Lebowitz J L, Maes C and Speer E R 1990 Statistical mechanics of probabilistic cellular automata J. Stat. Phys. 59 117-68

[9] Lebowitz J L, Maes C and Speer E R 1990 Probabilistic cellular automata: some statistical mechanical consderations 1989 Lectures in Complex Systems, SFI Studies in the Sciences of Complexity, Lecture Volume $I I$ ed $\mathrm{E}$ Jen (New York: Addison Wesley)

[10] He Y, Jayaprakash C and Grinstein G 1990 Generic behavior in locally interacting continuous systems Phys. Rev. A 42 3348-55

[11] Bramson M and Gray L 1991 A useful renormalization argument Festschrift for F. Spitzer (Birkhauser) in press

[12] van Beijeren H and Nolden I 1987 The roughening transition Topics in Current Physics, Structure and Dynamics of Surfaces $I I$ vol 43, ed W Schommers and P von Blanckenhagen (New York: Springer)

[13] Kandel D and Domany E 1990 Rigorous derivation of domain growth kinetics without conservation laws J. Stat. Phys. 58 685-706

[14] Hohenberg P C and Halperin B I 1977 Theory of dynamic critical phenomena Rev. Mod. Phys. $49435-69$

[15] Kardar M, Parisi G and Zhang Y C 1986 Dynamic scaling of growing interfaces Phys. Rev. Lett. 56 889-92

[16] Krug J and Spohn H 1991 Kinetic roughening of growing surfaces Solids Far from Equilbrium ed C Godreche (Cambridge: Cambridge University Press)

[17] Forster D, Nelson D R and Stephen M J 1977 Large-distance and long-time properties of a randomly stirred fuid Phys. Rev. A 16 732-49

[18] van Beijeren H, Kutner R and Spohn H 1985 Excess noise for driven diffusive systems Phys. Rev. Lett. $542026-9$

[19] Durrett R 1988 Lecture Notes on Particle Systems and Percolation (Pacific Grove, CA: Wadsworth and Brooks/Cole)

[20] Lebowitz J L and Speer E R 1991 in preparation

[2i] Derrida B and Dickman R 1991 On the interface between two growing Eden clusters J. Phys. A: Math. Gen. 24 L191-5 